\title{
Beam characterization and feasibility study for a small animal irradiation platform at clinical proton therapy facilities
}

\author{
S Gerlach ${ }^{1 *}, \mathbf{M}$ Pinto $^{1 *}, \mathbf{N}$ Kurichiyanil ${ }^{1 *}, \mathbf{C}$ Grau $^{2,3}, \mathbf{J}$ \\ Hérault $^{4,5}$, M Hillbrand ${ }^{6}$, PR Poulsen ${ }^{2,3}$, S Safai ${ }^{7}$, JM

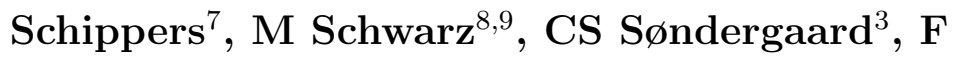 \\ Tommasino $^{8,10}$, Enrico Verroi $^{8}, \mathbf{M ~ V i d a l}{ }^{4,5}$, I Yohannes ${ }^{6}$, J \\ Schreiber $^{1}$ and K Parodi ${ }^{1}$ \\ * Authors contributed equally \\ ${ }^{1}$ Department for Medical Physics, Ludwig-Maximilians-Universität München, \\ Am Coulombwall 1, 85748 Garching, Germany \\ ${ }^{2}$ Department of Oncology, Aarhus University Hospital, Denmark \\ ${ }^{3}$ Danish Center for Particle Therapy, Aarhus University Hospital, Denmark \\ ${ }^{4}$ Centre Antoine Lacassagne, Nice, France \\ ${ }^{5}$ Fédération Claude Lalanne-Université Côte d'Azur, France \\ ${ }^{6}$ Rinecker Proton Therapy Center, München, Germany \\ ${ }^{7}$ Paul Scherrer Institute, Villigen, Switzerland \\ ${ }^{8}$ Trento Institute for Fundamental Physics and Applications, National Institute \\ for Nuclear Physics, Povo, Italy \\ ${ }^{9}$ Protontherapy Department, Azienda Provinciale per i Servizi Sanitari, Trento, \\ Italy \\ ${ }^{10}$ Department of Physics, University of Trento, Povo, Italy \\ E-mail: marco.pinto@physik.uni-muenchen.de
}

June 2020

Abstract. A deeper understanding of biological mechanisms to promote more
efficient treatment strategies in proton therapy demands advances in preclinical
radiation research. However this is often limited by insufficient availability of
adequate infrastructures for precision image guided small animal proton irradiation.
The project SIRMIO aims at filling this gap by developing a portable image-guided
research platform for small animal irradiation, to be used at clinical facilities and
allowing for a precision similar to a clinical treatment, when scaled down to the
small animal size. This work investigates the achievable dosimetric properties of 
different lowest energy clinical proton therapy beams, manipulated by a dedicated portable beamline including active focusing after initial beam energy degradation and collimation.

By measuring the lateral beam size in air close to the beam nozzle exit and the laterally integrated depth dose in water, an analytical beam model based on the beam parameters of the clinical beam at the Rinecker Proton Therapy Center was created for the lowest available clinical beam energy. The same approach was then applied to estimate the lowest energy beam model of different proton therapy facilities, Paul Scherrer Institute, Centre Antoine Lacassagne, Trento Proton Therapy Centre and the Danish Centre for Particle Therapy, based on their available beam commissioning data. This comparison indicated similar beam properties for all investigated sites, with emittance values of a few tens of $\mathrm{mm} \cdot \mathrm{mrad}$. Finally, starting from these beam models, we simulated propagation through a novel beamline designed to manipulate the beam energy and size for precise small animal irradiation, and evaluated the resulting dosimetric properties in water. For all investigated initial clinical beams, similar dosimetric results suitable for small animal irradiation were found.

This work supports the feasibility of the proposed SIRMIO beamline, promising suitable beam characteristics to allow for precise preclinical irradiation at clinical treatment facilities.

This is the version of the article before peer review or editing, as submitted to Physics in Medicine and Biology. IOP Publishing Ltd is not responsible for any errors

or omissions in this version of the manuscript or any version derived from it. The Version of Record is available online at https://doi.org/10.1088/1361-6560/abc832

\section{Introduction}

Radiation therapy with protons or light ions offers the potential for higher dose conformity compared to the more established photon therapy (Suit et al. 1982). While the technological progress over the last decades has increased the accuracy in proton therapy by enabling the delivery of highly conformal dose distributions making use of intensity modulated proton beams (Liu et al. 2012), the biological effects of radiation on the microenvironment of healthy and tumour tissues are not yet completely understood (Durante 2014).

Preclinical in vivo experiments are expected to help elucidating those open questions. While research platforms for image-guided small animal irradiation with photons are commercially available (e.g. the Small Animal Radiation Research Platform SARRP, 
Verhaegen et al. (2018)), similar systems are rare for proton beams. Although small animal irradiation with protons has already been performed without image guidance at research accelerators (e.g. Greubel et al. (2011)) and at clinical facilities (e.g. Müller et al. (2019), Bijl et al. (2002), Takata et al. (2015), Kondo et al. (2015), Moyers et al. (2007)), it is difficult to provide the desired small lateral beam size and precision in depth. More recently, Ford et al. (2017) and Kim et al. (2019) presented set-ups for small animal irradiation using the on-board X-ray cone beam computed tomography (CT) of the SARRP for image guidance. While the cyclotron used by Ford et al is capable of delivering beam energies lower than clinical ones, Kim et al used a cyclotron delivering clinical proton beam energies only down to $70 \mathrm{MeV}$. Therefore, a range shifter at the end of the beamline is used to degrade the beam energy to be suitable for small animal irradiation. In both cases, beam sizes suitable for small animal irradiation are achieved using a collimation system in close vicinity of the small animal. Also using a passive beam degradation and collimation system, in Beyreuther et al. (2018) a set-up for in vitro experiments dedicated to research in radiation biology is presented, which was subsequently extended to allow for in vivo studies.

The project SIRMIO (Small Animal Proton Irradiator for Research in Molecular Image-guided Radiation-Oncology, Parodi et al. (2019)) aims at building a portable image-guided irradiation platform for precise small animal irradiation to be used at different clinical proton therapy centres. In terms of image-guidance, the foreseen set-up includes proton transmission imaging and ultrasonic tumor localization for treatment planning and position verification along with on site range verification using ionoacoustics and positron-emission-tomography. This work aims to investigate the achievable dosimetric properties of a proposed new beamline concept which combines a movable degrader and collimator with active magnetic focusing (hereafter referred to as active beamline). To this end, a method to derive an analytical beam model from experimental data is used. Applying this method, beam models are obtained using experimental data of gantries and fixed beamlines from different clinical facilities to serve as input for beamline transport studies for SIRMIO. The necessary data are acquired in experiments at the Rinecker Proton Therapy Center (RPTC, gantry) in Munich in the scope of this work. Additionally, commissioning data are provided by the Paul Scherrer Institute (PSI, gantry), Centre Antoine Lacassagne (CAL, gantry), Trento Proton Therapy Centre (APSS, fixed beamline) and the Danish Centre for Particle Therapy (DCPT, gantry). We focus on these 
cyclotron- and synchrocyclotron-based facilities of different type and vendors as they typically exhibit higher beam intensity and a broader energy spectrum than lowest energie beams produced by synchrotrons (e. g. the Heidelberger IonenstrahlTherapiezentrum, Parodi et al. (2012)). The dosimetric evaluation of the beams manipulated by the proposed active beamline demonstrates the ability to produce beam energies and sizes which can allow for precise small animal irradiation. It is expected that the active beamline benefits from advantages in terms of entranceto-peak dose ratio and transmission compared to a purely passive design, besides avoiding to place highly activated material (e.g. collimators) and producing substantial neutron background in proximity to the biological target, as examined in Parodi et al. (2019).

\section{Materials and methods}

\subsection{Analytical model of the beam phase space}

For a realistic simulation of a proton beam travelling through an active beamline, an appropriate description of the incoming clinical beam is necessary. An analytical beam model for beams with Gaussian spatial and momentum distribution is explained in this section, following the approach given in Hinterberger (2008), Wiedemann (1993) and Carey (1987). The method solely relies on easily measurable dosimetric quantities - the lateral beam size at several positions in air and the laterally integrated depth dose (IDD) in water, along with the knowledge of the approximate initial beam energy. This makes the method being employable at any clinical facility and is here applied to experimental data acquired in this work at the RPTC as well as to similar data shared by different proton therapy centres. The modelling explained below can be separated in two parts: the spatial and angular distribution as well as the energy distribution. The complete analytical modelling is described in more detail in the Appendix A.

2.1.1. Spatial and angular distribution The beam broadening in x-direction along the propagation direction $z$ can be described in terms of sigma by

$$
\sigma_{\mathrm{x}}(z)=\sqrt{\sigma_{11}(0)+2 z \cdot \sigma_{12}(0)+z^{2} \cdot \sigma_{22}(0)} .
$$

Thereby $\sigma_{11}(0), \sigma_{12}(0)$ and $\sigma_{22}(0)$ are the beam parameters describing the variance of the spatial and angular distributions and their correlation, respectively, at $z=0$ 
which corresponds to the position of the last element in the beamline. To determine three unknowns, the beam sizes $\sigma_{\mathrm{x}}(z)$ need to be measured at least at three positions $z$. However, knowledge of the sigmas at more positions along the beam propagation direction reduces the uncertainties. The measured beam profiles include scattering due to air which contributes to the broadening of the beam. This scattering is assumed to induce a Gaussian lateral spread at different depths $z$ of the beam with the standard deviation $\sigma_{\text {air }}(z)$, which is systematically removed from the measured beam width $\sigma_{\mathrm{x}, \exp }(z)$ by

$$
\sigma_{\mathrm{x}}(z)=\sqrt{\sigma_{\mathrm{x}, \exp }(z)^{2}-\sigma_{\mathrm{x}, \mathrm{air}}(z)^{2}}
$$

In this work, the contribution of scattering in air is extracted from a Geant4 MC simulation (version 10.04.p02 Agostinelli et al. (2003)) of an infinitely narrow parallel beam in air for each beam energy. More details on the influence of scattering in air can be found in Appendix A.2. A fitting of the scatter-corrected beam sigmas $\sigma_{\mathrm{x}}(z)$ according to equation 11 yields the beam parameters as the fit parameters. The beam emittance describing the size of the beam ellipse in phase space can then by calculated by

$$
\epsilon_{\mathrm{x}}=\sqrt{\sigma_{11} \sigma_{22}-\sigma_{12}^{2}}
$$

The determination of the beam parameters in y-direction works correspondingly. Once the beam parameters are determined, they are used to sample horizontal and vertical phase spaces from Gaussian density distributions. More information on the modelling of the spatial and angular distribution is given in Appendix A.1.

2.1.2. Energy distribution The energy distribution of a clinical proton beam is approximated by a Gaussian distribution. The mean energy $E_{0}$ and the energy spread $\sigma_{\mathrm{E}_{0}}$ is deduced from a measurement of the IDD in a medium, e.g. water. Comparing the $R 80$ position ( $R 80$ being the position of the dose fall-off to $80 \%$ ) of the measured curve to the $R 80$ value of a calculated beam with the approximate mean energy (e.g. the nominal energy $E_{\text {nom }}$ which serves as an identifier for this beam in the clinical workflow and approximates the mean energy), the mean energy of the beam can be calculated as

$$
E_{0}=E_{\text {nom }}\left(\frac{R 80_{\text {exp }}}{R 80_{\text {nom }}}\right)^{1 / p}
$$


using $p=1.77$ (Bortfeld 1997). The final energy spread at the Bragg peak $\sigma_{\mathrm{E}_{\mathrm{f}}}$ is composed of two parts: the initial energy spread of the beam given by $\sigma_{\mathrm{E}_{0}}$ added up quadratically to the energy straggling induced by the interaction of the protons with the medium $\sigma_{\mathrm{E}_{\mathrm{S}}}$. To deduce the initial energy spread, a method developed by Bortfeld (1997) and Grassberger et al. (2015) is adapted for this purpose and explained in more detail in the appendix. A look up table is created with the peak widths $R 80-P 80$ ( $P 80$ being the position of the dose rise to $80 \%$ ) obtained from Geant4 simulations of proton beams with the nominal energy of interest as mean energy $E_{0}$ and different initial energy spreads $\sigma_{\mathrm{E}_{0}}$. The peak width in dependence of the energy spread is fitted using a second order polynomial as implied in Bortfeld (1997). The measured peak width is compared to the look up table and the corresponding energy spread can be determined. The energy of a single particle is then sampled from a one-dimensional Gaussian function with the determined mean energy $E_{0}$ and energy spread $\sigma_{\mathrm{E}_{0}}$. The correlation between energy and other phase space coordinates cannot be determined using this method, and is hence considered to be independent, which is a reasonable assumption for clinical pencil beams. The method described in this section to deduce the energy distribution of the beam is further explained in the Appendix A.3.

2.1.3. Validation and employment of the analytical beam model The developed analytical beam model is validated by a Geant4 simulation, using the simulation parameters given in the Appendix A.2. To assess the modelling of the spatial and angular distribution, the sampled phase space is simulated from the position of the nozzle exit $z=0$ in air and evaluated at certain positions $z$ downstream the nozzle, which coincide with the positions of the measurement of the lateral beam profile. Thus the simulated lateral beam width defined by the analytical beam model is compared to the experimentally determined width. Additionally, the phase space is simulated in water and the resulting IDD is compared to the experimental one to validate the modelled energy spectra.

In a similar way, the phase space is employed for the assessment of the SIRMIO beamline. The set-up described in section 3.3 is modelled in a Geant4 simulation and the phase space representing the analytical beam model is propagated from the position $z=0 \mathrm{~mm}$, which is the position of the nozzle exit. After travelling through the beamline, the beam propagates into a water phantom allowing for dosimetric evaluation. This procedure enables the assessment of the proposed beamline with respect to the validated and analytical beam model. 


\subsection{Experimental characterization of the RPTC lowest energy clinical beam}

Experiments were performed at the RPTC in Munich, Germany, at a beam energy of $75 \mathrm{MeV}$ and gantry angles $0^{\circ}$ (vertical) and $90^{\circ}$ (horizontal) in order to obtain a beam model as explained in section 2.1. The isocentre is $725 \mathrm{~mm}$ downstream of the last element in the beamline, which is the last multi strip ionization chamber (MSIC) in air. This position $z=0 \mathrm{~mm}$ is named nozzle exit. The lateral beam profiles are measured downstream the beam nozzle, for a gantry angle of $0^{\circ}$ at $z=305,525,725,825,925 \mathrm{~mm}$ and for a gantry angle of $90^{\circ}$ at $z=385,485,585,725,865,1005 \mathrm{~mm}$ using a scintillation screen coupled to a charge-coupled device (CCD, BIS ${ }^{2 \mathrm{G}}$,Wellhöfer-Scanditronix, nowadays IBA Dosimetry, Schwarzenbruck, Germany) with a pixel size of $0.37 \mathrm{~mm} \times 0.37 \mathrm{~mm}$. For $0^{\circ}$ gantry angle, the detector is placed directly on the patient table. For $90^{\circ}$ gantry angle, a special frame is used which is shown together with the detection system in figure $1 \mathrm{a}$ ). The background signal is subtracted from the measured data and a median filter with a kernel size of $3 \times 3$ pixels is applied to reduce signal enhancements caused by random image noise (e.g. scattered neutrons). To determine the beam sigmas, the transversal, two dimensional (2D) beam profile is laterally integrated in $x$ - and $y$-direction, to obtain a one dimensional (1D) profiles in $y$ - and $x$-direction with high statistics, respectively. Thereby $x$ - and $y$-direction refer to the axes of the room coordinate system, which are very slightly tilted against the major and minor axes of the spatial beam ellipse for the considered beam energy. A single Gaussian is fitted to the 1D profile, from which the standard deviation is obtained as beam sigma in the corresponding direction.

To measure the IDD, a water phantom (PTW MP3-XS Phantom Tank, PTW Freiburg, Germany) is used, in which a parallel plate ionization chamber is inserted on a motorized stage (PTW Bragg Peak Chamber 34070). In figure 1 b) the setup is shown. The measured data are corrected to account for the water equivalent thickness (WET) of the entrance window of the Bragg peak chamber and the window of the reference ionization chamber positioned at the entrance of the phantom, which are $4.05 \mathrm{~mm}$ and $0.25 \mathrm{~mm}$, respectively. The resolution in beam direction is $0.1 \mathrm{~mm}$ in the Bragg peak region, defined by the step size of the moving ionization chamber. 


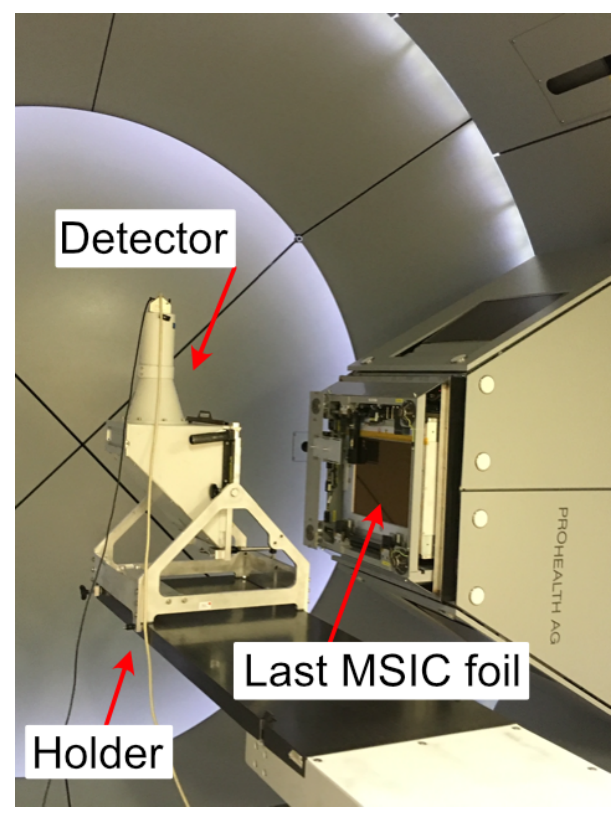

(a)

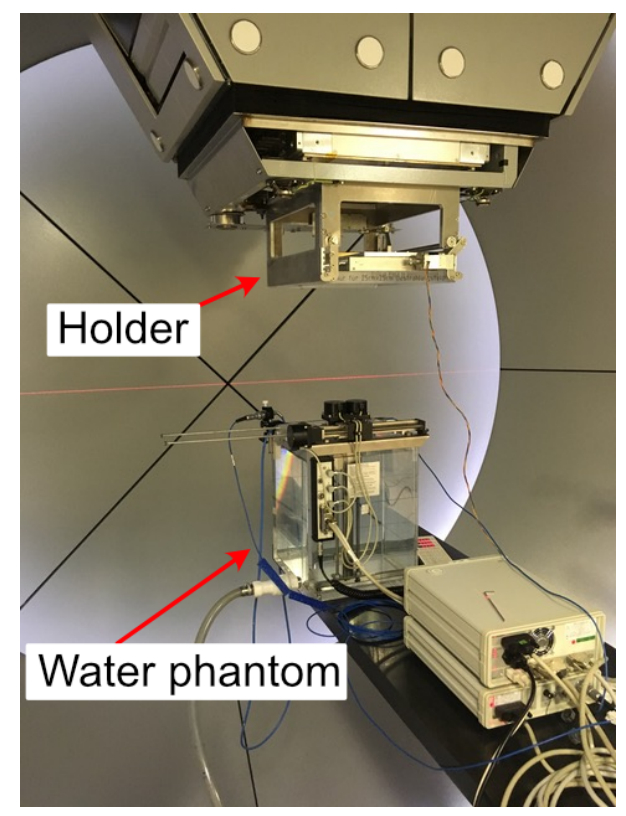

(b)

Figure 1: a) Set-up for the detection of the lateral beam profile in air at RPTC with a gantry angle of $90^{\circ}$ using a scintillation detector. The detector is placed with a special holder on the patient table. The last MSIC foil is visible in the beam nozzle. b) Water phantom with an ionization chamber to measure the laterally integrated depth dose profile of the beam at a gantry angle of $0^{\circ}$. A holder for the potential placement of a degrader is hanging from the nozzle (not used for the IDD measurement).

\subsection{Characterization and comparison of different lowest energy clinical proton beams}

Beam characterization according to the analytical beam model described in section 2.1 is carried out for five European proton therapy facilities, which are briefly introduced in the following. All facilities use a cyclotron or synchrocyclotron to accelerate ions to the maximal available energy, along with a subsequently placed degrader to reduce the beam energy. This procedure defines the lowest available clinical beam energy, which is reported in this section. If in the clinical workflow even lower beam energies are needed, the facilites are equipped with an additional range shifter, which can be positioned in the nozzle close to the patient. The RPTC 
in Munich, Germany, was built by ACCEL (nowadays Varian Medical Systems, Inc., Palo Alto, California) and is equipped with a superconducting cyclotron accelerating protons up to a nominal energy of $250 \mathrm{MeV}$. The degrader positioned behind the cyclotron allows for clinically available beam energies ranging from $245 \mathrm{MeV}$ to $75 \mathrm{MeV}$. The facility has four equivalent treatment rooms, each equipped with a gantry including the one where beam characterization is done in this work, and one fixed beam room initially foreseen for ocular treatment. The CAL in Nice, France, hosts two protontherapy rooms. One room is dedicated to ocular treatments and related to a dedicated $65 \mathrm{MeV}$ cyclotron. The other room is a single room solution with a gantry and the superconducting synchrocyclotron S2C2 developed by IBA which provides protons accelerated up to $230 \mathrm{MeV}$. The lowest clinical beam energy available with $\mathrm{S} 2 \mathrm{C} 2$ at this facility is $100 \mathrm{MeV}$ (Kleeven et al. 2013). A beam model has been set-up for the gantry beamline in this study. Similar to the RPTC, the PSI in Villingen, Switzerland, is equipped with a superconducting cyclotron developed by ACCEL, capable of delivering protons with nominal energies ranging from $70 \mathrm{MeV}$ to $250 \mathrm{MeV}$. Besides one fixed beamline, three gantries are available, two of which are in clinical use (PSI 2019) and one is dedicated to research. In this study, a beam model is set-up for the beam exiting gantry 2. The APSS is the proton therapy centre in Trento, Italy. The Proteus 235 cyclotron developed by IBA delivers beam energies between $70 \mathrm{MeV}$ and $228 \mathrm{MeV}$ to two treatment rooms equipped with a gantry and one fixed beamline, for which beam characterization is performed (Tommasino et al. 2017). Finally, a model is developed for the proton beam at the DCPT in Aarhus, Denmark, using data from the gantry, which is equivalent to the beam transported by the fixed beamline. Acceleration is done with a cyclotron provided by Varian delivering beam energies ranging from $70 \mathrm{MeV}$ to $250 \mathrm{MeV}$. Additionally, three treatment rooms equipped with a gantry are available for patient treatment (PTCOG 2019).

For the purpose of this study, in addition to own measurements performed at the RPTC (section 2.2) experimental data were shared by the corresponding facilities. These data included the IDD in water as well as the beam width in air at five positions downstream the nozzle exit. 
2.4. In-silico dosimetric characterization of lowest energy clinical beams transported by the SIRMIO beamline

The proton beam from a clinical facility has to be degraded in energy, collimated, transported and refocused in order to meet the requirements of SIRMIO as a small animal irradiation platform. To this end, several beamline options consisting of degraders, collimators and a triplet of permanent magnet quadrupoles were simulated in vacuum. In this work, the results obtained in a Geant4 simulation study using an active beamline design (Kurichiyanil et al. 2019) sketched in figure 2 are presented for all considered facilities. It includes a graphite degrader to obtain beams with

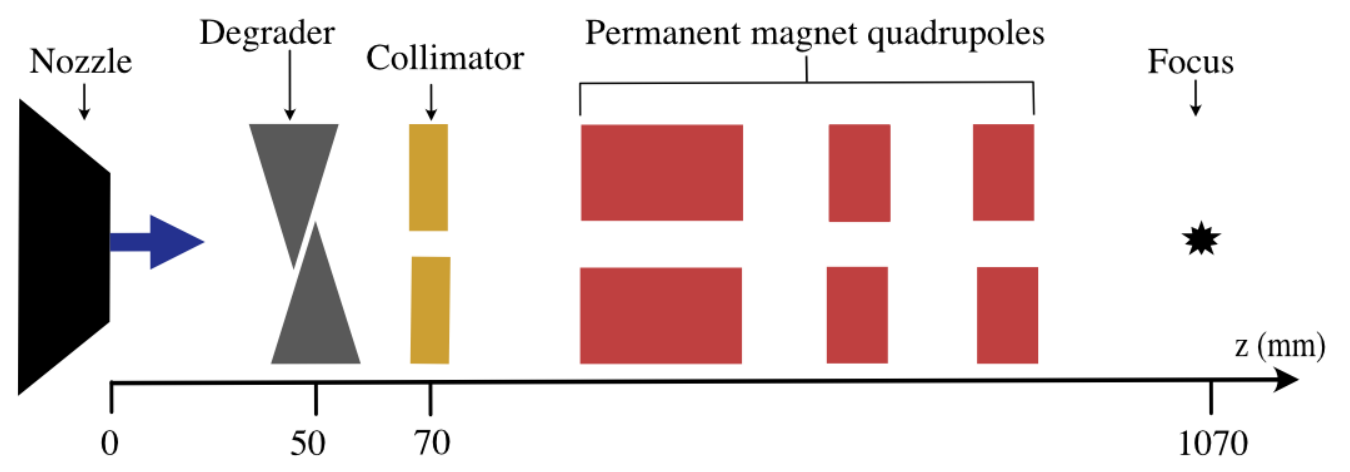

Figure 2: Schematic drawing of the active beamline consisting of a degrader (graphite), a collimator (brass) and a triplet of permanent magnet quadrupoles $(\mathrm{NdFeB})$. The beam is entering from the nozzle, indicated with a blue arrow.

energies ranging from 20 to $60 \mathrm{MeV}$ from a clinical beam. It is positioned $500 \mathrm{~mm}$ downstream the nozzle exit. The nominal initial beam energies vary from 70 to $100 \mathrm{MeV}$, depending on the facility, as listed in table 1. A brass collimator of $40 \mathrm{~mm}$ thickness with a square opening of $1.2 \mathrm{~mm} \times 1.2 \mathrm{~mm}$ scales the emittance of the beam to match the acceptance of the subsequent triplet of permanent magnets. The magnets are optimized in order to focus the range of beam energies to beam sizes of $1.6 \mathrm{~mm}$ full width at half maximum (FWHM) at the focus. The position of the magnets along the beam axis $z$ is adjusted depending on the beam energy being focused to keep the focal point at a fixed position. Not shown in the schematic drawing is the water phantom with a size of $5 \mathrm{~cm} \times 5 \mathrm{~cm} \times 10 \mathrm{~cm}$ and a binning size of $50 \mu \mathrm{m} \times 50 \mu \mathrm{m} \times 50 \mu \mathrm{m}$ for the evaluation of dosimetric quantities in the simulations. 
It is positioned such that the focus of the manipulated proton beam coincides with the Bragg peak location. From the simulated IDD, the entrance-to-peak ratio (EPR) and the tail-to-peak ratio (TPR) is calculated by

$$
\mathrm{EPR}=\frac{D_{\text {entrance }}}{D_{\text {peak }}} \quad \mathrm{TPR}=\frac{D_{\text {tail }}}{D_{\text {peak }}},
$$

with $D_{\text {entrance, }} D_{\text {peak }}$ and $D_{\text {tail }}$ being the laterally integrated dose at the entrance of the water phantom, at the Bragg peak and at the dose tail $(3 \mathrm{~mm}$ behind the peak), respectively, each of those averaged in depth over five bins. Furthermore the peak width $(R 80-P 80)$ and the distal fall-off $(R 20-R 80)$ of the IDD is calculated. The FWHM and the lateral penumbra (lateral dose fall-off from $80 \%$ to $20 \%$ ) are calculated from the lateral beam profile at the focus.

\section{Results}

\subsection{Experimental characterization of the RPTC lowest energy clinical beam}

As an example, the lateral beam profile of the $75 \mathrm{MeV}$ RPTC beam measured at the isocentre $(z=725 \mathrm{~mm})$ in air at a gantry angle of $0^{\circ}$ is shown in figure $\left.3 \mathrm{a}\right)$. Figure $3 \mathrm{~b})$ shows the profile data laterally integrated in $y$, along with the Gaussian fit. The small spots of relatively high signal visible in figure 3 a) are random image noise which is being reduced by a median filter applied to the acquired data prior to the fitting of the data with a Gaussian function. Especially at the centre of the distribution the fit shows good agreement with the data, which leads to small uncertainties of the beam sigma estimation. For both investigated gantry angles, the lateral beam sigma in $x$ - and $y$-direction is shown for all measured positions in figure $4 \mathrm{a}$ ) and b), respectively. The uncertainties resulting from the Gaussian fit of the lateral beam profile are always smaller than $0.3 \%$ and are too small to be visible in the plots. It can be seen that the gantry angle has a clear influence on the beam size, resulting in differences of $0.5 \mathrm{~mm}$ (in $x$ - and $y$-direction) at the isocentre, while only a small impact on the beam divergence.

The measured beam sigmas and the data after applying corrections for beam broadening due to scattering in air are shown in figure 5 for the measurement at a gantry angle of $0^{\circ}$ degree. A fit using equation 1 is shown in the same figure and the beam parameters resulting from the fit are given in table 1. These beam parameters are used to model a phase space, from which protons are sampled 


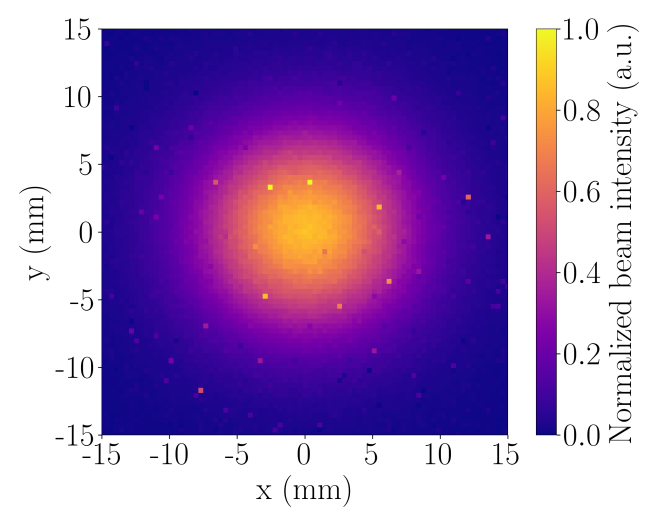

(a)

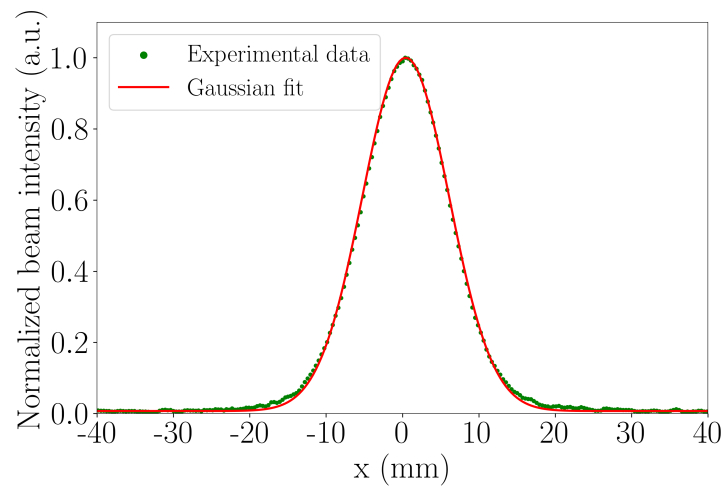

(b)

Figure 3: a) Experimentally determined two dimensional intensity profile in the $x / y$ plan of the proton beam at RPTC at the isocentre.

b) In $y$-direction laterally integrated profile at the isocentre. The green dots are the median filtered experimental data, in red the Gaussian fit is shown.

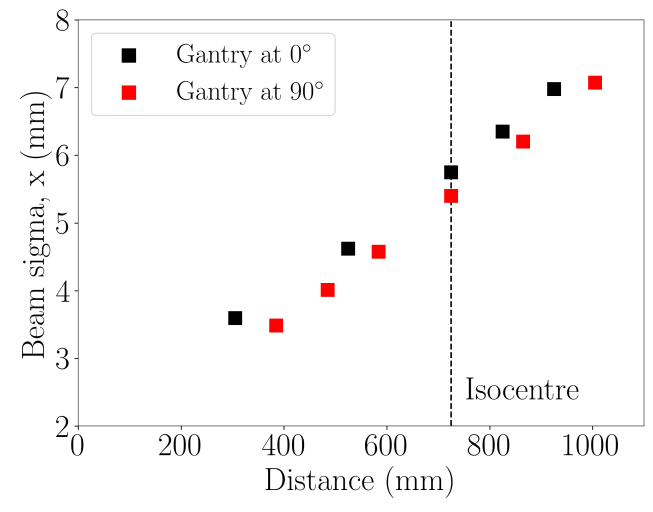

(a)

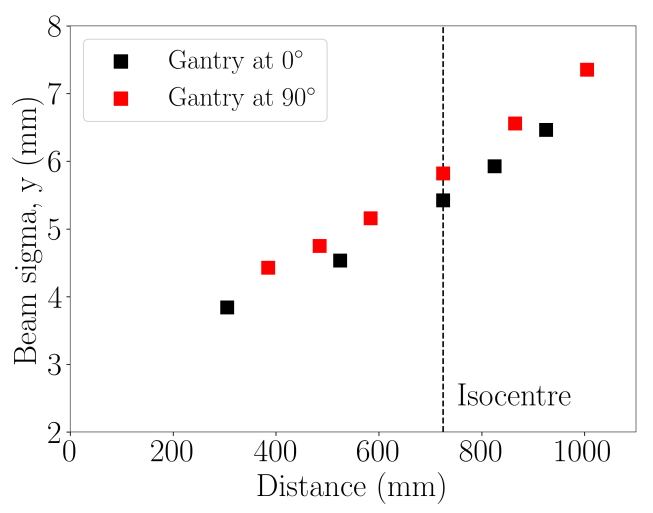

(b)

Figure 4: Lateral beam sigma of the proton beam measured at RPTC in a) $x$ - and b) $y$-direction along the beam propagation axis. The black squares correspond to a gantry angle of $0^{\circ}$, the red squares to gantry angle of $90^{\circ}$. Uncertainties are too small to be visible. 


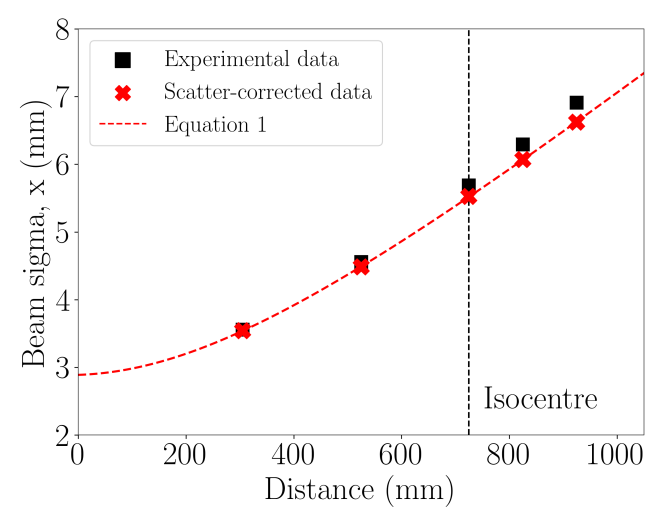

(a)

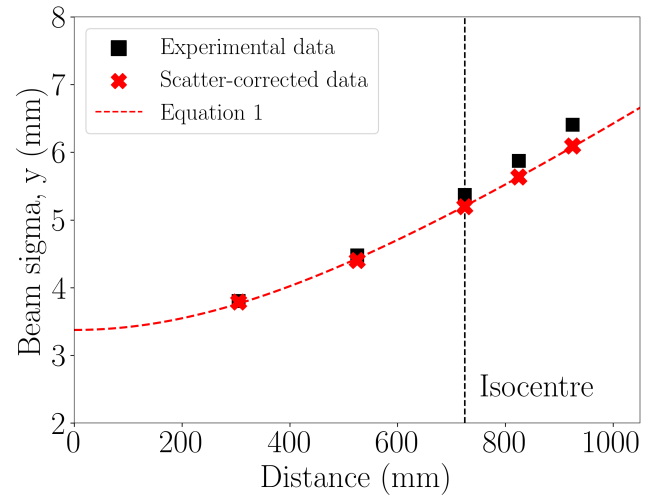

(b)

Figure 5: Illustration of the scattering correction applied to the measured data. The black squares correspond to the experimentally determined beam size at a gantry angle of $0^{\circ}$ in a) $x$ - and b) $y$-direction at five positions downstream the beam nozzle, whose last element is located at $0 \mathrm{~mm}$. The beam width corrected for scattering in air is shown with red crosses, the dashed red line corresponds to the fit according to equation 1 .

and propagated through air and water to cross verify the beam profiles and IDD, respectively, between experiment and simulation. The comparisons of the profiles are shown in figure 6a) and 6b). Fitting errors are less than $0.3 \%$ and not visible in the figures. The analytical beam model reproduces the beam closely at every evaluated position. The simulated and experimentally measured IDDs are compared in figure 7. The error bars of the measured data points arise from the uncertainty of the motor moving the ionization chamber $(0.1 \mathrm{~mm})$. The uncertainty of the dose measurement is determined by the relative error between the dose measurement of the reference ionization chamber and the Bragg peak chamber, which is considered to be negligible. The $R 80$ position and the peak width are determined to be $46.02 \mathrm{~mm}$ and $1.96 \mathrm{~mm}$, respectively. An uncertainty of $0.40 \mathrm{~mm}$ is chosen for the uncertainty of the $R 80$ position, as this is the allowed tolerance which was met in several preceding measurements. Both profiles show overall good agreement with a difference in $R 80$ position below $0.1 \mathrm{~mm}$ due to a small deviation in mean energy $E_{0}$. 


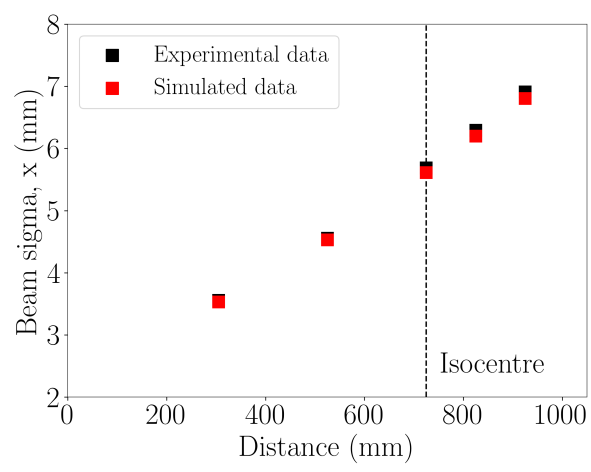

(a)

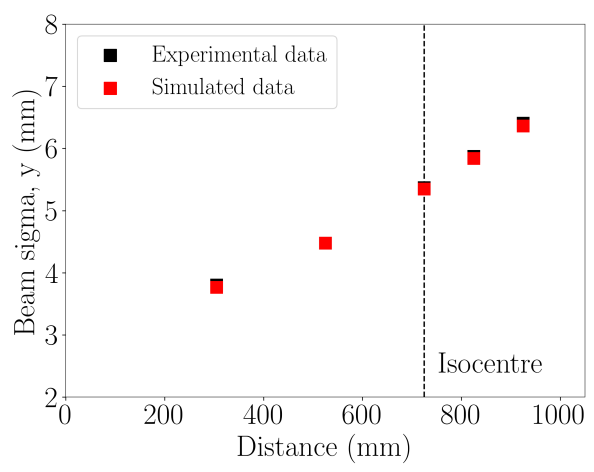

(b)

Figure 6: Lateral beam size of the proton beam at RPTC in a) $x$ - and b) $y$-direction at five positions downstream the nozzle exit. The black squares correspond to the measured data at a gantry angle of $0^{\circ}$. The red squares result from a Geant4 simulation using the analytical beam model.

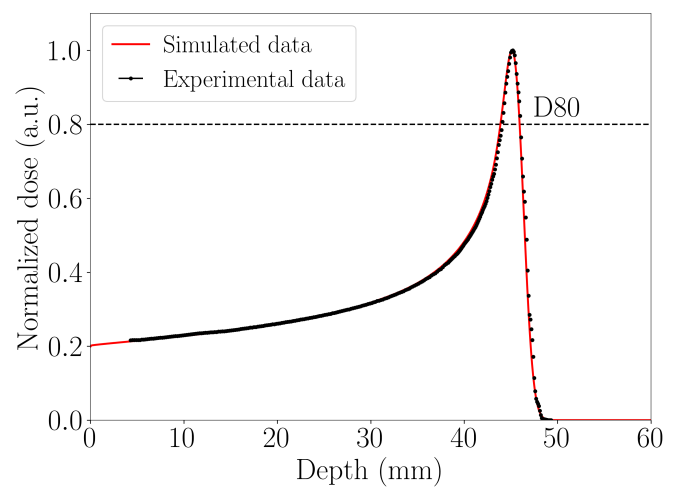

(a)

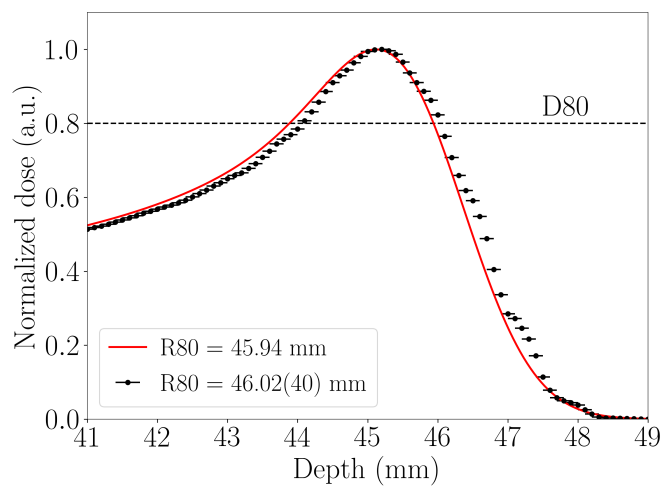

(b)

Figure 7: a) Normalized depth-dose profiles of the $75 \mathrm{MeV}$ RPTC beam in water. The black data points correspond to measured data with uncertainties, the red curve corresponds to the simulation using the developed beam model. Due to the WET of the ionization chamber and the reference chamber, the experimental data are only visible for depths larger than $4.3 \mathrm{~mm}$. For a better overview the dose fall-off to $80 \%$ is shown with a line marked by $D 80$. In b) a zoom of the profiles is shown to emphasize the agreement, along with the $R 80$ position. 


\subsection{Characterization and comparison of different lowest energy clinical proton} beams

Analytical beam models have been developed for all the investigated clinical beams, using experimental data provided by the facilities. This includes IDDs as well as beam widths in air at several positions downstream the nozzle, which suffered scattering in air. For the beamline at the APSS, the mean energy and the energy spread is taken from Tommasino et al. (2017) due to the too coarse resolution of the available IDDs. For all other available beams, this information is determined from the IDD as explained in section 2.1.2. The obtained model beam parameters along with the mean energy and the energy spread are presented in table 1. The given uncertainties arise from the analytical fitting procedure of the beam sigmas. Additionally, the maximal available beam current at the isocentre is given for the corresponding beam energy. For the RPTC $90^{\circ}$ gantry angle ( $x$-direction) and the DCPT (y-direction) the available data are measured too far from the beam waist, and therefore the fit function cannot describe the behaviour close to the nozzle. Therefore the beam parameter $\sigma_{\mathrm{x}}(0)$ for RPTC and $\sigma_{\mathrm{y}}(0)$ for DCPT are set to be $2.5 \mathrm{~mm}$ and $2.8 \mathrm{~mm}$, respectively, which allowed fitting of the data at other positions. The forcing of the fit parameters in these cases leads to relatively high uncertainties of the beam parameters. The values reported for the PSI gantry beamline refer a fully retracted nozzle. The full extraction of the nozzle influences the beam parameters, leading to a decrease in emittance of approx. $10 \%$. From the reported intensities, there is a clear trend of larger beam currents at fixed beamlines than gantries. For example, currents at the considered fixed beamlines can be up to $4.0 \mathrm{nA}$. 
Table 1: Beam parameters at the nozzle exits of the beams at the Rinecker Proton Therapy Center (RPTC), Paul Scherrer Institute (PSI), Centre Antoine Lacassagne (CAL), Trento Proton Therapy Centre (APSS) and the Danish Centre for Particle Therapy (DCPT). The beam parameters of the proton beam at the RPTC are determined at $0^{\circ}$ and $90^{\circ}$ gantry angles. For RPTC, the required data were experimentally determined as explained in section 2.2 for all other cases the data were shared by the corresponding facilities as discussed in section 2.3 .

${ }^{1}$ Gantry ${ }^{2}$ Fixed beamline $\quad *$ Current at the fixed beamline

\begin{tabular}{llclclc}
\hline & ${ }^{1} \mathrm{RPTC}-0^{\circ}$ & ${ }^{1} \mathrm{RPTC}-90^{\circ}$ & ${ }^{1} \mathrm{CAL}$ & ${ }^{1} \mathrm{PSI}$ & ${ }^{2}$ APSS & ${ }^{1} \mathrm{DCPT}$ \\
\hline $\mathrm{E}_{\mathrm{nom}}(\mathrm{MeV})$ & 75 & 75 & 100 & 70 & 70 & 70 \\
$\mathrm{E}_{0}(\mathrm{MeV})$ & 75.32 & & 100.45 & 71.16 & 68.5 & 71.41 \\
$\sigma_{\mathrm{E}_{0}}(\mathrm{MeV})$ & 0.58 & & 0.83 & 0.55 & 0.48 & 0.88 \\
\hline$\sigma_{\mathrm{x}}(\mathrm{mm})$ & $2.8(1)$ & $2.5(2)$ & $4.8(1)$ & $4.0(1)$ & $4.3(1)$ & $4.2(5)$ \\
$\sigma_{\mathrm{xx}^{\prime}}(\mathrm{mm} \cdot \mathrm{mrad})$ & $1.9(2)$ & $1.0(36)$ & $5.4(3)$ & $6.9(2)$ & $-6.9(13)$ & $-2.1(54)$ \\
$\sigma_{\mathrm{x}^{\prime}}(\mathrm{mrad})$ & $6.2(1)$ & $6.0(4)$ & $5.5(1)$ & $8.1(1)$ & $4.8(1)$ & $5.2(6)$ \\
$\epsilon_{\mathrm{x}}(\mathrm{mm} \cdot \mathrm{mrad})$ & $17.5(2)$ & $14.8(18)$ & $25.9(4)$ & $18.0(6)$ & $19.4(10)$ & $21.6(38)$ \\
\hline$\sigma_{\mathrm{y}}(\mathrm{mm})$ & $3.6(1)$ & $3.9(1)$ & $4.2(1)$ & $4.7(1)$ & $2.0(3)$ & $2.8(5)$ \\
$\sigma_{\mathrm{yy}^{\prime}}(\mathrm{mm} \cdot \mathrm{mrad})$ & $-1.8(4)$ & $-1.6(1)$ & $10.3(10)$ & $-0.6(2)$ & $-0.4(21)$ & $3.0(62)$ \\
$\sigma_{\mathrm{y}^{\prime}}(\mathrm{mrad})$ & $5.8(1)$ & $6.0(1)$ & $5.6(2)$ & $8.4(1)$ & $4.7(2)$ & $5.0(7)$ \\
$\epsilon_{\mathrm{y}}(\mathrm{mm} \cdot \mathrm{mrad})$ & $20.4(1)$ & $23.4(5)$ & $21.2(11)$ & $39.7(2)$ & $9.3(16)$ & $13.9(48)$ \\
\hline Intensity $(\mathrm{nA})$ & 0.4 & & 0.4 & 0.3 & 0.6 & $0.2\left(4.0^{*}\right)$ \\
\hline
\end{tabular}


From table 1 it is also evident that small differences in the beam sizes and divergences are present between beams provided by different facilities. However, an upstream collimator redefines the phasespace accepted by the beamline to the central part of the beam phasespace. This is visualized in figure 8 in which the beam ellipse of the RPTC beam is shown at the position of the collimator together with the collimator acceptance. For this visualization, the beam model is propagated analytically from the beam nozzle to the position of the collimator, contrary to the simulation of the particles propagating from the nozzle through the SIRMIO beamline, for which a Geant4 simulation is used as explained in section 2.4. Protons which are not in the intersection of both areas, cannot travel through the collimator. Therefore it can be concluded that the considered beams are suitable to be used with the SIRMIO beamline, but with corresponding variation in transported fraction of the beam. The phase space in figure 8 does not take into account the influence of the degrader positioned upstream the collimator. However, it is located very close to the collimator and therefore has a minor influence on the beam width at this position.

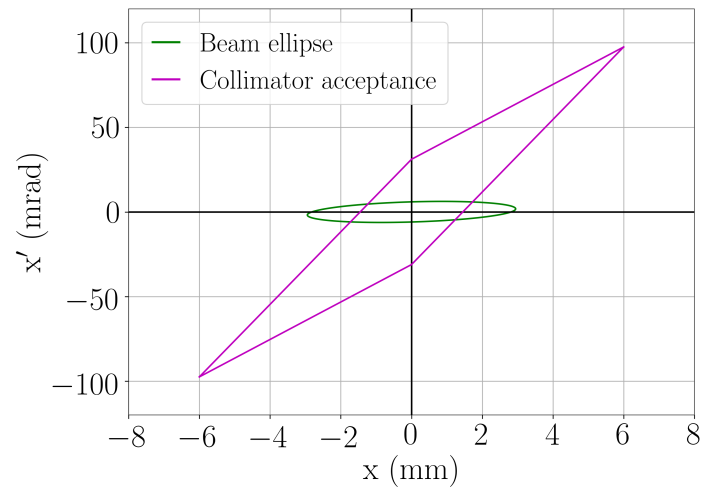

(a)

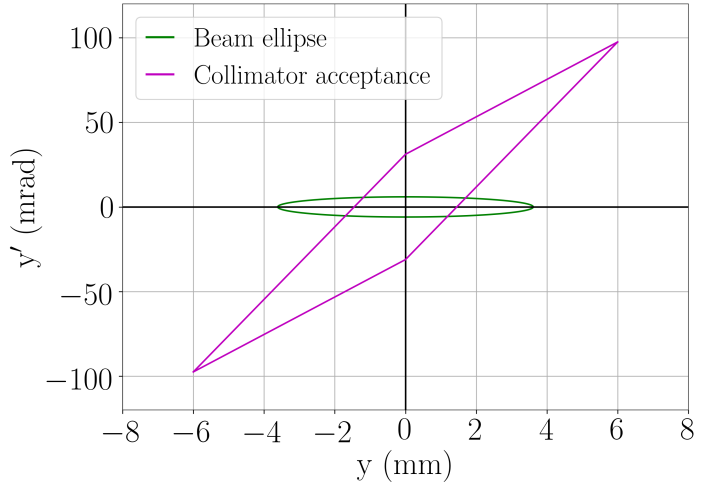

(b)

Figure 8: Beam ellipse (black) of the RPTC beam in a) $x$-and b) $y$-direction at the position of the collimator. The acceptance of the collimator is shown in magenta. 
3.3. In-silico dosimetric characterization of lowest energy clinical beams transported by the SIRMIO beamline

The simulated IDDs of the clinical proton beams transported and manipulated by the SIRMIO beamline in water are shown in figure 9 exemplarily for beam energies degraded to $20 \mathrm{MeV}$ and $50 \mathrm{MeV}$, starting from the modelled phase space of the respective facilities at the initial proton beam energies of table 1. The Bragg peaks

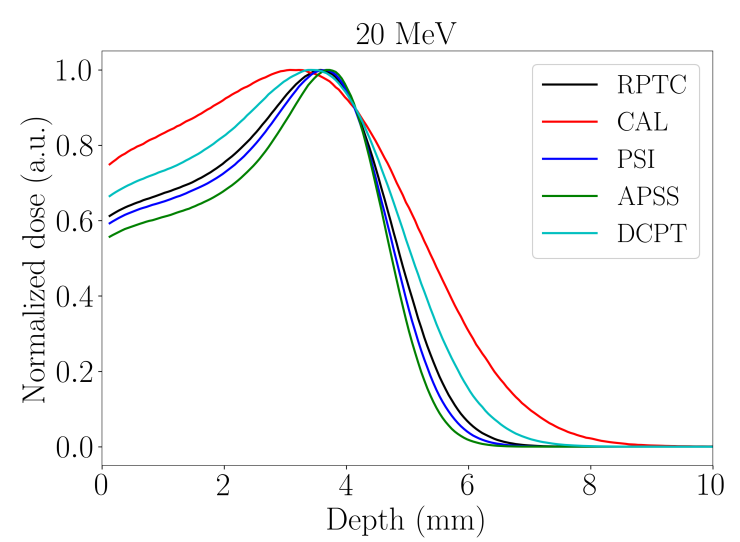

(a)

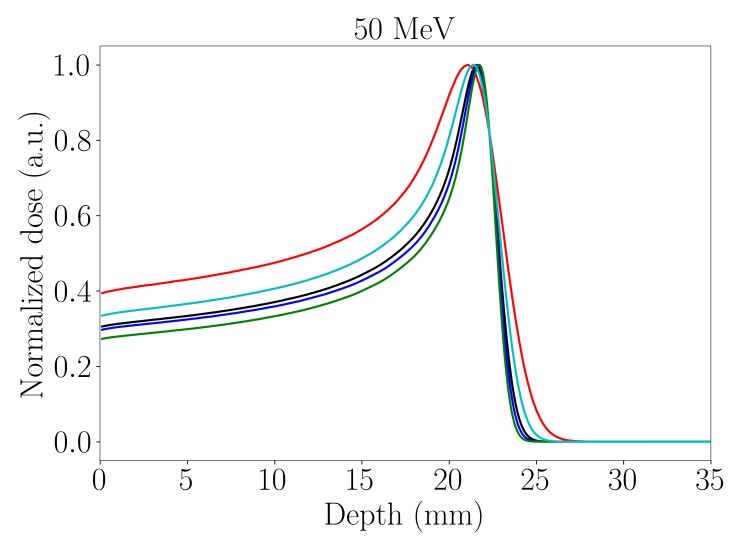

(b)

Figure 9: Geant4 simulated depth dose profiles in water of the a) $20 \mathrm{MeV}$ and b) $50 \mathrm{MeV}$ proton beams obtained with the presented beamline, starting from the analytical models of the lowest energy clinical beams at the corresponding colourcoded facilities.

are located at $3.4 \mathrm{~mm}$ and $21.4 \mathrm{~mm}$, respectively. Between the different simulated clinical beams small deviations are observable, which result in an uncertainty of $\pm 0.5 \mathrm{~mm}$ in range. To further evaluate and assess the longitudinal beam properties, fundamental parameters (defined in section 2.2) are calculated and displayed in figure 10. In general, it is expected that beams which are subject to more degradation show poorer performance than beams which experience less local degradation, as this increases the energy spread. This can be seen in figure 10 in case of the CAL beam with an initial energy of $100.45 \mathrm{MeV}$. The dose in the entrance region is higher compared to the other beams, the peak width is larger and also the distal dose falls slower. However, also the initial spread of the beam energy influences these results. 


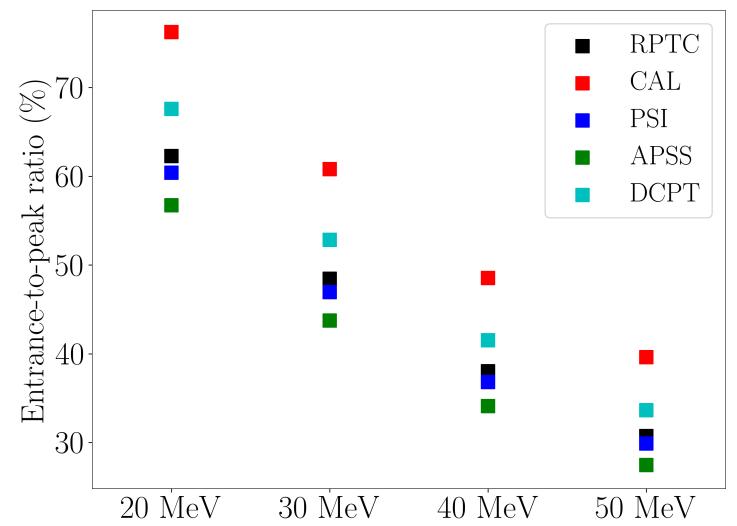

(a)

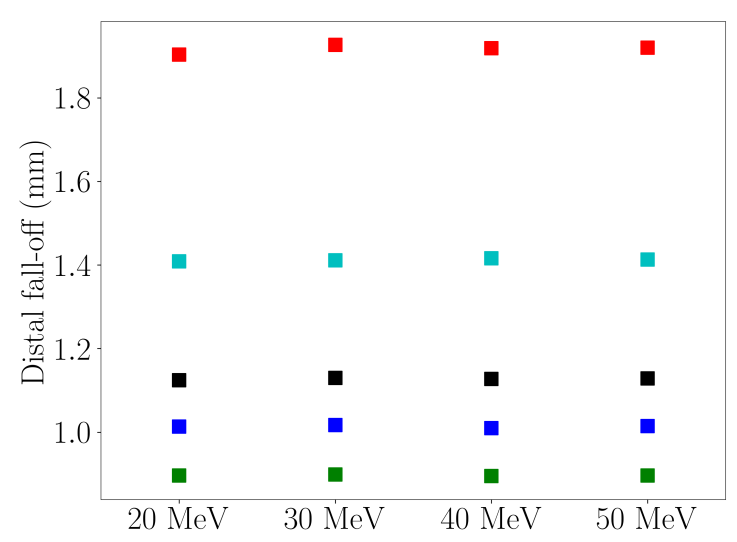

(c)

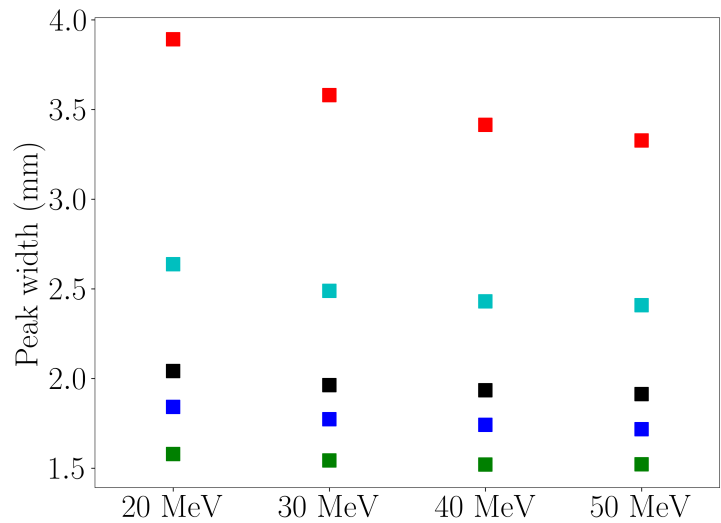

(b)

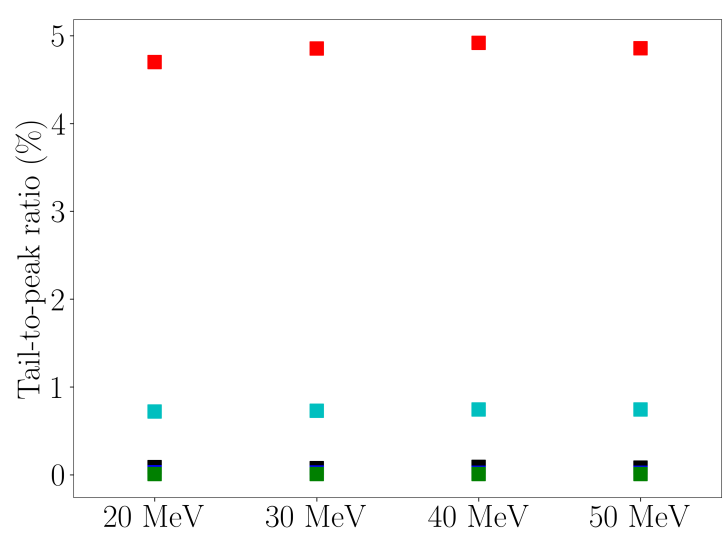

(d)

Figure 10: a) Entrance-to-peak ratio, b) peak width $(R 80-P 80)$, c) distal fall-off $(R 20-R 80)$ and d) tail-to-peak ratio of the energy degraded and actively focused proton beams for four beam energies. The color code displayed in figure a) indicates the facility of which the beam model was used as input file.

Likewise, the APSS beam (fixed experimental beamline) with the lowest initial beam energy of $68.5 \mathrm{MeV}$ and the smallest initial energy spread of $0.48 \mathrm{MeV}$ leads to the best results in terms of EPR, peak width, distal fall-off and TPR. When comparing the beams with respect to their final energy, the EPR and the peak width is reduced towards increasing energies.

The lateral dose profile in $x$-direction at the focus of the manipulated beams is 
shown in figure 11 for energies degraded to $20 \mathrm{MeV}$ and $50 \mathrm{MeV}$. The corresponding

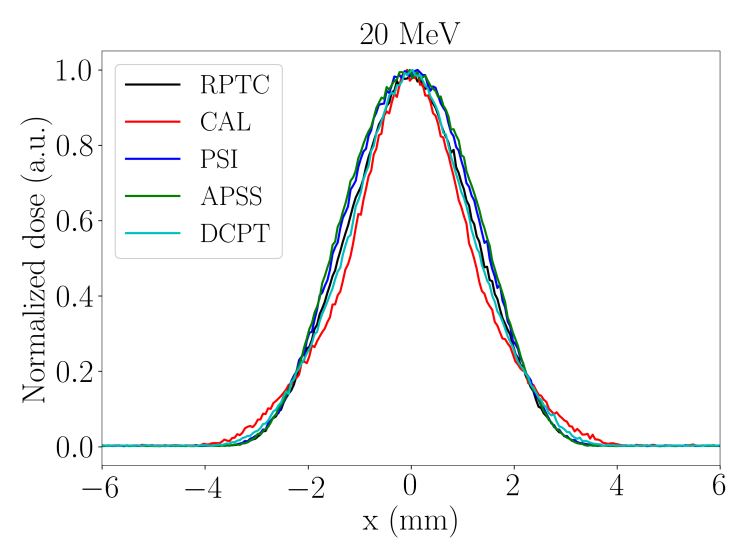

(a)

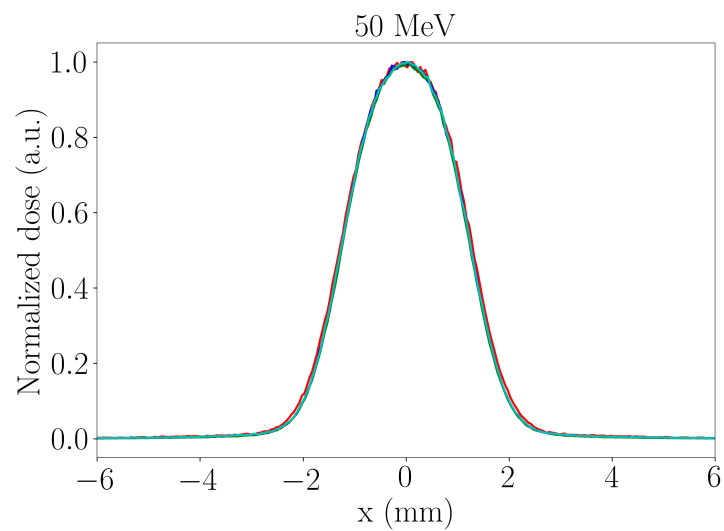

(b)

Figure 11: Normalized lateral dose profiles of the manipulated beams degraded to energies of a) $20 \mathrm{MeV}$ and b) $50 \mathrm{MeV}$ at the focus. The color code indicates the facility of which the beam model was used as input file.

lateral penumbra and the FWHM are shown in figure 12 for both profiles in $x$ and $y$-direction. For the profile in $x$-direction, the manipulated beams of $20 \mathrm{MeV}$ show differences up to $0.3 \mathrm{~mm}$ for the lateral penumbra and $0.7 \mathrm{~mm}$ for the FWHM, depending on the incident clinical beam. For all other beam energies, only small differences below $0.06 \mathrm{~mm}$ (lateral penumbra) and $0.3 \mathrm{~mm}$ (FWHM) are found. In $y$-direction, differences up to $0.4 \mathrm{~mm}$ for the lateral penumbra and $1.0 \mathrm{~mm}$ for the FWHM were observed for all the beam energies. All clinical beams show the smallest lateral beam size when being degraded to $30 \mathrm{MeV}$. With values of approximately $0.5 \mathrm{~mm}$ and $0.7 \mathrm{~mm}$, the APSS beam shows the smallest penumbra in $x$ - and $y$ direction, respectively, along with the smallest FWHM of $1.1 \mathrm{~mm}$ and $1.3 \mathrm{~mm}$.

The energy spread of the manipulated beam is influenced by the extent of degradation along with the initial energy spread. For further assessment, the energy spread of the transported beam is extracted from the simulation and fitted with a single Gaussian and the sigma $\sigma_{\mathrm{Ef}}$ is extracted in dependence of the final beam energy and the facility of which the beam model was used as input file. The result is shown in figure 13. The influence of the degradation process on the final energy spread is visible as increasing energy spread with decreasing mean energy of the focused 


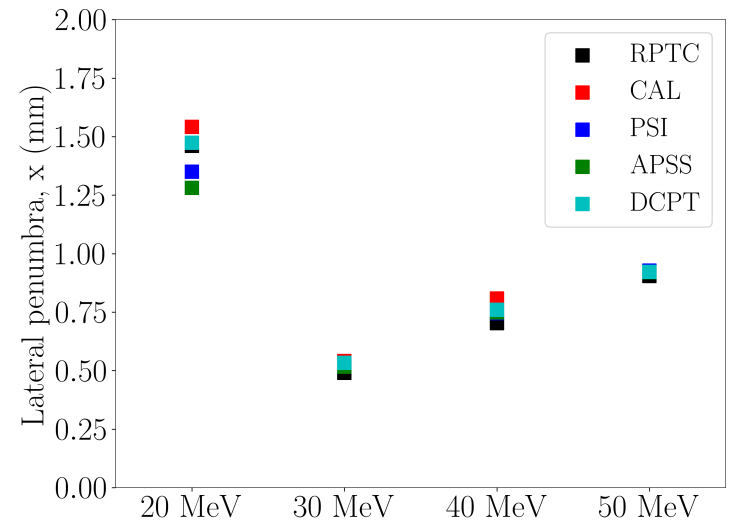

(a)

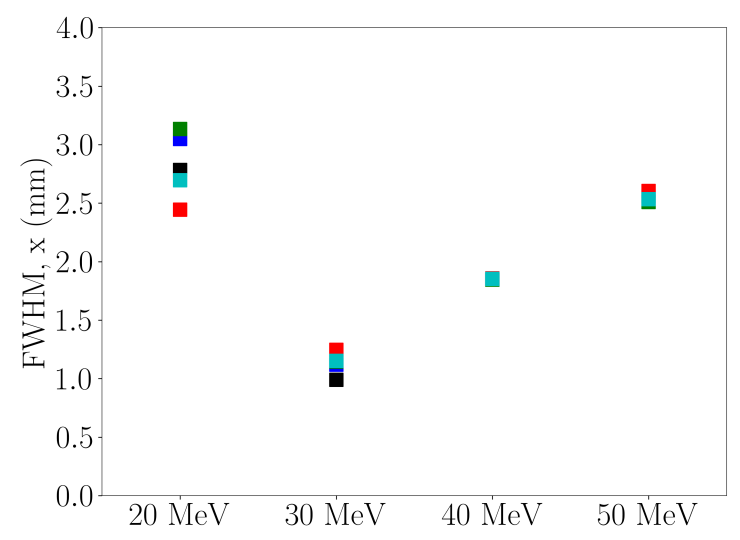

(c)

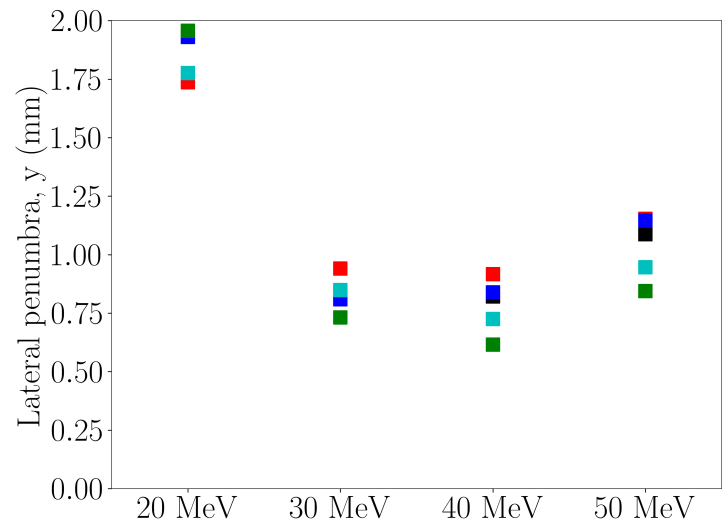

(b)

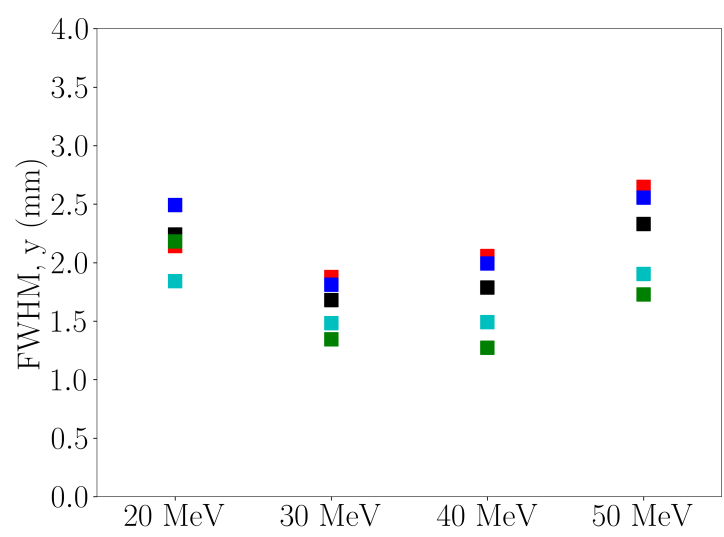

(d)

Figure 12: Lateral penumbra (80\% to $20 \%$ ), top, in a) $x$ - and b) $y$-direction along with full width at half maximum (FWHM), bottom, in a) $x$ - and b) $y$-direction of the transported beams at the focus for four investigated beam energies. The color code indicates the facility of which the beam model was used as input file.

beams. Differences in energy spread of the focused beams arising from differences in initial beam energies and energy spreads are visible when degraded beams using beam models of different facilities are examined, however, more studies are needed to evaluate the dependency on the initial energy spread. This is particularly emphasized for the comparison between CAL and APSS. 


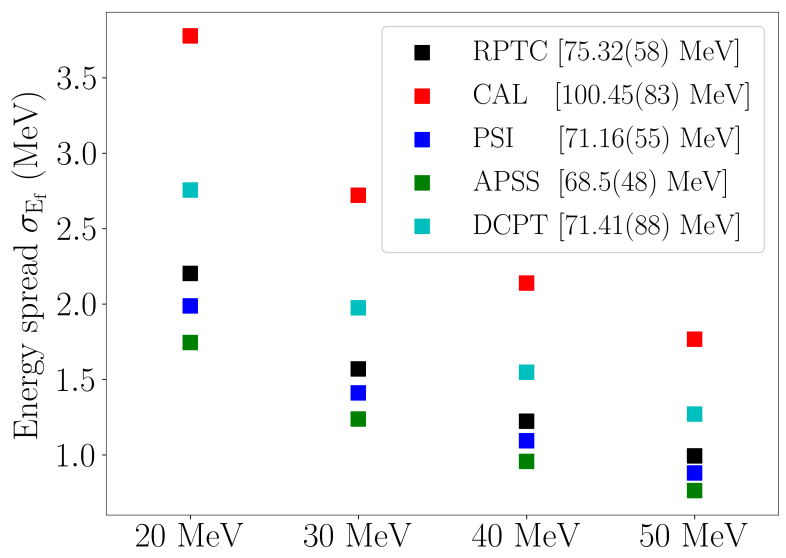

Figure 13: Energy spread in terms of $\sigma_{\mathbf{E}_{\mathrm{f}}}$ of the manipulated beam after passing the permanent magnet quadruplet for different final beam energies. The colorcode indicates the facility of which the beam model was used as input file. Additionally, the mean energy of the incoming clinical beam is given in brackets for the perspective facility.

\section{Discussion}

The project SIRMIO aims at the development of a portable research platform enabling precise and image-guided small animal irradiation at clinical facilities. This demands the development of a beamline which is able to modify clinical proton beams in energy and size for small animal irradiation. In this study, beam models of five European facilities were created based on measured local beam characteristics and transported through a novel beamline composed of degrading, collimating and focusing elements in a simulation study. The results show overall similar properties of the manipulated beams, which confirms the feasibility of a portable beamline concept. The details of the beamline are still under optimization and will be reported in a separate study together with the final assessment of the manipulated beam properties for small animal irradiation in terms of treatment planning.

In the past five years, two systems were proposed for image-guided in-vivo research in proton therapy by Ford et al. (2017) and Kim et al. (2019). Ford et al show in a simulation study that the degradation of a $100 \mathrm{MeV}$ to a $30 \mathrm{MeV}$ beam with an acrylic degrader results in an EPR larger than $50 \%$. In our study, this corresponds 
to the degradation of the beam at CAL showing an EPR of $61 \%$ in agreement to the findings of Ford et al. However, the EPR can be improved to less than $45 \%$ for a degraded beam of $30 \mathrm{MeV}$ when using the clinical beam of the APSS with a nominal initial energy of $70 \mathrm{MeV}$. Furthermore, in Ford et al measurements are performed with beams of energies down to $30 \mathrm{MeV}$ emerging from a cyclotron. The lateral beam size is varied using collimators of different sizes, resulting in a typical penumbra of $0.8 \mathrm{~mm}$. In this study the penumbra ranges from $0.5 \mathrm{~mm}$ to $2.0 \mathrm{~mm}$, which is in good agreement to the finding of Ford et al. Kim et al employ a clinical beam with an initial energy ranging from $77 \mathrm{MeV}$ to $98 \mathrm{MeV}$, hence a solid water plastic degrader of constant thickness is used to scale down the beam energy as well as two collimators are employed to reduce the lateral beam size. The energy of the beam from the cyclotron is varied to yield manipulated beams of different ranges. For evaluation, the ranges of the simulated beams of this study are calculated and compared to the ranges of the beams presented in Kim et al. Best agreement was found for the beams of $20 \mathrm{MeV}$ and $77 \mathrm{MeV}, 30 \mathrm{MeV}$ and $80 \mathrm{MeV}, 40 \mathrm{MeV}$ and $85 \mathrm{MeV}$ along with $50 \mathrm{MeV}$ and $89 \mathrm{MeV}$ (where for each pair the final energy of the SIRMIO beam is given together with the initial beam energy in Kim et al). However, in all cases the energy of the beam investigated in this study is lower than the energy of the corresponding beam in Kim et al. This holds especially for the beam with the lowest energy being $77 \mathrm{MeV}(R 90=5.96 \mathrm{~mm})$ which is compared to the manipulated beam of $20 \mathrm{MeV}$ in this study $(R 90=3.52 \mathrm{~mm})$. When choosing the fixed beam of the APSS as initial beam, a significant improvement is found for all final beam energies using the SIRMIO beamline with respect to the EPRs as defined in this study. As the SIRMIO beamline makes use of the lowest available clinical beam energy and varies the degrader thickness to yield different final beam energies, the amount of degrader material passed by the beam is minimized. This results in a manipulated beam with smaller energy spread, visible as a smaller EPR. For the beam of lowest energy (corresponding to a final energy of $20 \mathrm{MeV}$ in this study and an initial energy of $77 \mathrm{MeV}$ in the study of Kim et al) the IDDs show EPRs of $57 \%$ and approx. $65 \%$, respectively, for the beam of highest energy (corresponding to a final energy of $50 \mathrm{MeV}$ along with a $R 90$ of $21.46 \mathrm{~mm}$ in this study and an initial energy of $89 \mathrm{MeV}$ along with a $R 90$ of $21.68 \mathrm{~mm}$ in the study of Kim et al) EPRs of $27 \%$ and approximately $35 \%$ are found, respectively. In Kim et al, the lateral beam shape is modelled with a collimation system of different sizes. A beam width similar to the beams in this study is found for a collimator size of $3 \mathrm{~mm} \times 3 \mathrm{~mm}$. Such set-up 
results in a beam width of approx. $2 \mathrm{~mm}$ FWHM, which is in between the findings of this study with $1 \mathrm{~mm}$ to $3 \mathrm{~mm}$ FWHM. When comparing the presented active beamline to the design of a purely passive beamline developed in the course of this study (Parodi et al. 2019), advantages in transmission and a reduction of the neutron fluence close to the target are found.

In the process of the SIRMIO beamline design, a challenge was found when aiming simultaneously at a small spot size and a high transmission to guarantee suitable treatment times. These goals are conflicting, as a closing of the collimator opening to reduce the lateral beam size also reduces the particle fluence.

The next steps in this project will include the testing of the adapted clinical beams in terms of treatment planning. Furthermore, possible advantages of an additional range shifter behind the last element of the beamline are investigated in an ongoing study. This is motivated by two findings of this work. Firstly, the beamline presented in this study shows overall the best results for a beam degraded down to $30 \mathrm{MeV}$. Besides degrading the beam even further upstream, another option is to install a range shifter close to the focus. Secondly, preliminary treatment plans have shown the possible necessity of beam energies below $20 \mathrm{MeV}$ for selected murine tumour indications (Kundel 2019). This issue can also be addressed by the installation of an additional range shifter close to the target. Also not discussed in this work is the activation of the beamline. Especially for the portability of the beamline it is necessary that all components show low activity after being irradiated. This requires the development of a proper shielding system, which is being investigated in a separate study. A possible consequence is a relatively heavy set-up that must be linked to facility-specific constraints on the maximal permitted weight placeable in a treatment room. This favours the installation of SIRMIO in fixed beamlines of experimental rooms. Additionally, fixed beamlines typically show beam currents up to one order of magnitude higher than delivered by a gantry. This applies not only to the few experimental fixed beamlines considered in this study, where currents up to $4.0 \mathrm{nA}$ (DCPT) were reported, but also to dedicated ocular beamlines available e.g. at CAL, RPTC and PSI, where also currents up to $4.0 \mathrm{nA}$ can be expected. The SIRMIO set-up is especially intended for the former experimental fixed beamlines, usually dedicated to quality assurance and radiobiological experiments. The increased beam currents will be beneficial to counteract beam losses in the dedicated SIRMIO beamline. Considering future developments in relation to FLASH (Buonanno et al. 2019), (van de Water 
et al. 2019), beam currents are also expected to raise.

\section{Conclusion}

In this work, we demonstrated the capability of a proposed novel active beamline to be used with proton beams from different proton therapy centres for producing suitable beams for small animal irradiation. Analytical models of five clinical beams were created and validated. In a simulation study, these beams were transported through an active beamline leading to similar dosimetric results for all facilities. Beam sizes suitable for a small animal treatment with similar precision as a clinical treatment could be achieved. This paves the way for the realization of the project SIRMIO, aiming at a portable installation which can manipulate different lowest energy clinical beams for precise small animal irradiation.

\section{Acknowledgements}

This work is funded by the European Research Council (ERC) under the European Union's Horizon 2020 research and innovation programme through the grant agreement number 725539 . 


\section{Appendix A. Analytical model of the beam phase space}

The analytical beam model used for the description of clinical beams with Gaussian spatial and momentum distribution is explained in this section in more detail, following the approach given in Hinterberger (2008), Wiedemann (1993) and Carey (1987).

\section{Appendix A.1. Spatial and angular distribution}

In this derivation, the horizontal and vertical motions are considered to be decoupled, which allows treating the respective phase spaces independently from each other. Although the derivation of the spatial and angular beam properties is done for monoenergetic beams, it is valid for beams with small energy spread, such as usually observed for clinical proton beams.

A particle beam can be represented by an ensemble of points in the six-dimensional phase space, which is described by a density distribution $\rho\left(x, y, x^{\prime}, y^{\prime}, z, E\right)$ (Hinterberger 2008). For example the horizontal phase-space spanned by the position $(x)$ and divergence $\left(x^{\prime}\right)$ of the particles is considered for the derivation. This approach is equally applicable to the vertical phase space $\left(y, y^{\prime}\right)$. Most density distributions can be surrounded by an ellipse in phase space (Hinterberger 2008), described by

$$
\boldsymbol{x}^{\top} \boldsymbol{\sigma}_{\mathrm{x}}{ }^{-1} \boldsymbol{x}=1
$$

where $\boldsymbol{x}^{\top}=\left(x, x^{\prime}\right)$ is the beam vector pointing from the origin of the coordinate system to the edge of the beam ellipse. The covariance matrix for $x$ and $x^{\prime} \boldsymbol{\sigma}_{\mathrm{x}}$

$$
\boldsymbol{\sigma}_{\mathrm{x}}=\left(\begin{array}{ll}
\sigma_{11} & \sigma_{12} \\
\sigma_{21} & \sigma_{22}
\end{array}\right), \quad \sigma_{21}=\sigma_{12}
$$

is positive definite and symmetric, and its components $\sigma_{11}, \sigma_{22}$ and $\sigma_{12}$ are the variance of the spatial and angular distributions and their correlation, respectively. When using other definition of beam parameters as e.g. the doubled spatial variance, doubled covariance and doubled angular variance (Safai et al. 2008), care must be taken in conversion. Following Liouville's theorem, the area of the beam ellipse stays constant when the beam is transported through systems described by conservative forces (e.g. electromagnetic). The conserved area is known as beam emittance $\epsilon$ and can be derived from the covariance matrix. The emittance in $x$-direction is given by

$$
\epsilon_{\mathrm{x}}=\sqrt{\operatorname{Det}\left(\boldsymbol{\sigma}_{\mathrm{x}}\right)}=\sqrt{\sigma_{11} \sigma_{22}-\sigma_{12}^{2}}
$$


Assuming a Gaussian distribution of the beam intensity, the density function is described by the normalized-, two dimensional function

$$
\rho(\boldsymbol{x})=\frac{1}{2 \pi \epsilon_{\mathrm{x}}} \exp \left(-\frac{1}{2} \boldsymbol{x}^{\top} \boldsymbol{\sigma}_{\mathrm{x}}{ }^{-1} \boldsymbol{x}\right) .
$$

The density distribution is characterised by elliptic isoprobability contours. The beam ellipse defined in equation A.1 is the contour, in which the density is reduced to a factor of $\exp (-1 / 2)$ compared to the maximum density at the centre of the distribution. The maximal extension of the beam ellipse is given by one standard deviation of the spatial and the angular distributions of the particles. In $x$-direction, these quantities correspond to

$$
\sigma_{\mathrm{x}}=\sqrt{\sigma_{11}} \quad \sigma_{\mathrm{x}^{\prime}}=\sqrt{\sigma_{22}} .
$$

The correlation between $\sigma_{\mathrm{x}}$ and $\sigma_{\mathrm{x}^{\prime}}$

$$
\sigma_{\mathrm{xx}^{\prime}}=\sigma_{12}
$$

describes the orientation of the elliptical axes. The case of $\sigma_{12}<0$ corresponds to a focusing beam and $\sigma_{12}>0$ corresponds to a diverging beam. The location of the minimal lateral beam width is called beam waist.

The transformation of the beam parameters from an initial position $z=0$ to any other position $z$, where $z$ is the axis of beam propagation, is described by the action of the transfer matrix $\boldsymbol{R}$ as

$$
\boldsymbol{\sigma}_{\mathrm{x}}(z)=\boldsymbol{R}_{\mathrm{x}}(z) \boldsymbol{\sigma}_{\mathrm{x}}(0) \boldsymbol{R}_{\mathrm{x}}(z)^{\top}
$$

(Penner 1961). The transfer matrix $\boldsymbol{R}_{\mathrm{x}}(z)$ is found by solving the equations of motion for the elements in the beamline. For the purpose of this work, the relevant part is the beam propagating downstream from the last element of the beamline. To describe this, the transfer matrix for a free drift in vacuum given by

$$
R_{\mathrm{drift}}=\left(\begin{array}{ll}
1 & z \\
0 & 1
\end{array}\right)
$$

is thus sufficient. The influence of scattering in air is considered in a separate Monte Carlo study, as explained in section Appendix A.2. Solving equation A.7 with equation A.8 yields

$$
\sigma_{\mathrm{x}}(z)=\sqrt{\sigma_{11}(z)}=\sqrt{\sigma_{11}(0)+2 z \cdot \sigma_{12}(0)+z^{2} \cdot \sigma_{22}(0)}
$$

where $\sigma_{11}(0), \sigma_{12}(0)$ and $\sigma_{22}(0)$ are the beam parameters at $z=0 \mathrm{~mm}$. To determine three unknowns, the beam sizes need to be measured at least at three positions. 
However, knowledge of the sigmas at more positions along the beam propagation direction reduces the uncertainties. A fitting of these data according to equation A.9 yields the beam parameters as the fit parameters. Once the beam parameters are determined, they are used to sample horizontal and vertical phase spaces from respective multivariate Gaussian density function given by equation A.4.

\section{Appendix A.2. Modelling of the scattering in air}

The measured beam profiles include scattering due to air which contributes to the broadening of the beam. This scattering contribution has to be systematically removed from the measured beam profiles in order to correctly estimate the beam sigmas at the nozzle exit. This scattering is assumed to induce a Gaussian lateral spread at different depths $z$ of the beam with the standard deviation $\sigma_{\text {air }}(z)$. The position dependent beam width $\sigma_{\mathrm{x}}(z)$ can be determined by correcting the measured width $\sigma_{\mathrm{x}, \exp }$ using

$$
\sigma_{\mathrm{x}}(z)=\sqrt{\sigma_{\mathrm{x}, \exp }(z)^{2}-\sigma_{\mathrm{x}, \mathrm{air}}(z)^{2}}
$$

As an example, using Highland's formula (Highland 1975) the influence of scattering in air can be estimated to introduce a beam broadening of $\sigma_{\mathrm{x}, \text { air }}(z)=1.5 \mathrm{~mm}$ at the isocentre of the RPTC set-up. In this work, the contribution of scattering in air is extracted from a MC simulation of an infinitely narrow parallel beam in air for each beam energy. The simulation toolkit Geant4 (version 10.04.p02 Agostinelli et al. (2003)) is used with the physics models being the ones from the reference physics list QGSP_BIC_HP but replacing the default emstandard_opt3 with the more accurate emstandard_opt4 for the modeling of electromagnetic physics, along with an air density of $0.00120479 \mathrm{~g} / \mathrm{cm}^{3}$ and an ionization potential of $85.7 \mathrm{eV}$ (Berger et al. 1984). This approach is more accurate than using Highland's formula since the latter takes into account only the Gaussian part of multiple Coulomb scattering and therefore neglects other contributions to the lateral spreading of a beam such as large angle scattered protons caused by interactions with nuclei (Pedroni et al. 2005) which influence the fitting of a single Gaussian function to the data.

\section{Appendix A.3. Energy distribution}

In this work, the modelling of clinical beams with Gaussian energy distribution is considered. The mean energy $E_{0}$ and the energy spread $\sigma_{\mathrm{E}_{0}}$ is deduced from a 
measurement of the IDD in a medium, e.g. water. The IDD is measured for a clinical beam with a certain nominal energy $E_{\text {nom }}$, which labels this beam in the clinical workflow and approximates the mean energy of the beam. To determine the mean energy $E_{0}$, the polynomial expression

$$
R_{0}=\alpha E_{0}^{p}
$$

is used with $R_{0}$ being the range in the medium. The parameters $\alpha$ and $p$ depend on the absorbing medium and the energy, respectively (Newhauser \& Zhang 2015). Comparing the $R 80$ position ( $R 80$ being the position of the dose fall-off to $80 \%$ ) of the measured curve to the $R 80$ value of a calculated beam with the approximate mean energy (e.g. the nominal energy) and an arbitrary energy spread (on which the $R 80$ value does not depend (Schuemann et al. 2014)), the mean energy of the beam can be calculated as

$$
E_{0}=E_{\text {nom }}\left(\frac{R 80_{\exp }}{R 80_{\text {nom }}}\right)^{1 / p}
$$

using $p=1.77$ (Bortfeld 1997).

The correlation between IDD and energy spread is more complex. Proton dose calculation algorithms typically rely on Monte Carlo simulations of the beam nozzle and measured IDD curves (Hong et al. 1996), (Würl et al. 2016). However, a simpler and faster analytical model developed by Bortfeld (1997) and further investigated by Grassberger et al. (2015) was adopted for this purpose. The final energy spread at the Bragg peak $\sigma_{\mathrm{E}_{\mathrm{f}}}$ is composed of two parts: the initial energy spread of the beam given by $\sigma_{\mathrm{E}_{0}}$ added up quadratically to the energy straggling induced by the interaction of the protons with the medium $\sigma_{\mathrm{ES}_{\mathrm{S}}}$

$$
\sigma_{\mathrm{E}_{\mathrm{f}}}^{2}=\sigma_{\mathrm{E}_{\mathrm{S}}}^{2}+\sigma_{\mathrm{E}_{0}}^{2} .
$$

The polynomial relationship between range and energy given in equation A.11 is used for the translation of the standard deviation of the energy to the standard deviation of the range straggling. To describe the range straggling caused by the initial energy spread of the beam, equation A.11 is linearized around $E_{0}$. This is valid under the assumption that the initial energy spread is small compared to the mean energy, i. e. $\sigma_{\mathrm{E}_{0}} \ll E_{0}$. The standard deviation of the range distribution $\sigma_{\mathrm{R}_{f}}$ can then be described by

$$
\sigma_{\mathrm{R}_{\mathrm{f}}}^{2}=\sigma_{\mathrm{R}_{\mathrm{S}}}^{2}+\sigma_{\mathrm{E}_{0}}^{2}\left(\frac{\mathrm{d} R_{0}}{\mathrm{~d} E_{0}}\right)^{2}=\sigma_{\mathrm{R}_{\mathrm{S}}}^{2}+\sigma_{\mathrm{E}_{0}}^{2} \alpha^{2} p^{2} E_{0}^{2 p-2},
$$


with $\sigma_{\mathrm{R}_{\mathrm{S}}}$ being the standard deviation of the range for a mono-energetic beam. A look up table is created with the peak widths $R 80-P 80$ ( $P 80$ being the position of the dose rise to $80 \%$ ) obtained from simulations of proton beams with the nominal energy of interest as mean energy $E_{0}$ and different initial energy spreads $\sigma_{\mathrm{E}_{0}}$. Only the $P 80$ value depends on the energy spread, however peak width are reported to make this method robust against small changes in mean energy. The simulation toolkit Geant4 is used with the water density set to be $0.99777 \mathrm{~g} / \mathrm{cm}^{3}$ (corresponding to a room temperature of $22^{\circ}$ degree) and an ionization potential of $78.0 \mathrm{eV}$ (Seltzer et al. 2014). The nominal energies of interest are 70,75 and $100 \mathrm{MeV}$ in this work, corresponding to the lowest clinical beam energies at the considered facilities. The simulated initial energy spreads range from 0.0 to $1.2 \mathrm{MeV}$ in steps of $0.2 \mathrm{MeV}$ in order to cover expected energy spreads in a clinical beam. The peak width in dependence of the energy spread is fitted using a second order polynomial as implied by equation A.14. The measured peak width is compared to the look up table with simulated peak widths and the corresponding energy spread can be determined. The energy of a single particle is then sampled from a one-dimensional Gaussian function with the determined mean energy $E_{0}$ and energy spread $\sigma_{\mathrm{E}_{0}}$. The correlation between energy and other phase space coordinates cannot be determined using this method, and is hence considered to be independent, which is a reasonable assumption for clinical pencil beams. 


\section{References}

Agostinelli, S., Allison, J., Amako, K., Apostolakis, J., Araujo, H., Arce, P., Asai, M., Axen, D., Banerjee, S., Barrand, G., Behner, F., Bellagamba, L., Boudreau, J., Broglia, L., Brunengo, A., Burkhardt, H., Chauvie, S., Chuma, J., Chytracek, R., Cooperman, G., Cosmo, G., Degtyarenko, P., Dell'Acqua, A., Depaola, G., Dietrich, D., Enami, R., Feliciello, A., Ferguson, C., Fesefeldt, H., Folger, G., Foppiano, F., Forti, A., Garelli, S., Giani, S., Giannitrapani, R., Gibin, D., Gómez Cadenas, J., González, I., Gracia Abril, G., Greeniaus, G., Greiner, W., Grichine, V., Grossheim, A., Guatelli, S., Gumplinger, P., Hamatsu, R., Hashimoto, K., Hasui, H., Heikkinen, A., Howard, A., Ivanchenko, V., Johnson, A., Jones, F., Kallenbach, J., Kanaya, N., Kawabata, M., Kawabata, Y., Kawaguti, M., Kelner, S., Kent, P., Kimura, A., Kodama, T., Kokoulin, R., Kossov, M., Kurashige, H., Lamanna, E., Lampén, T., Lara, V., Lefebure, V., Lei, F., Liendl, M., Lockman, W., Longo, F., Magni, S., Maire, M., Medernach, E., Minamimoto, K., Mora de Freitas, P., Morita, Y., Murakami, K., Nagamatu, M., Nartallo, R., Nieminen, P., Nishimura, T., Ohtsubo, K., Okamura, M., O’Neale, S., Oohata, Y., Paech, K., Perl, J., Pfeiffer, A., Pia, M., Ranjard, F., Rybin, A., Sadilov, S., Di Salvo, E., Santin, G., Sasaki, T., Savvas, N., Sawada, Y., Scherer, S., Sei, S., Sirotenko, V., Smith, D., Starkov, N., Stoecker, H., Sulkimo, J., Takahata, M., Tanaka, S., Tcherniaev, E., Safai Tehrani, E., Tropeano, M., Truscott, P., Uno, H., Urban, L., Urban, P., Verderi, M., Walkden, A., Wander, W., Weber, H., Wellisch, J., Wenaus, T., Williams, D., Wright, D., Yamada, T., Yoshida, H. \& Zschiesche, D. (2003). Geant4 - a simulation toolkit, Nuclear Instruments and Methods in Physics Research Section A: Accelerators, Spectrometers, Detectors and Associated Equipment 506(3): 250-303.

Berger, M. J., Inokuti, M., Anderson, H. H., Bichsel, H., Dennis, J. A., Powers, D., Seltzer, S. M. \& Turner, J. E. (1984). Report 37, Journal of the International Commission on Radiation Units and Measurements os19(2): NP-NP.

Beyreuther, E., Baumann, M., Enghardt, W., Helmbrecht, S., Karsch, L., Krause, M., Pawelke, J., Schreiner, L., Schürer, M., von Neubeck, C. \& Lühr, A. (2018). Research Facility for Radiobiological Studies at the University Proton Therapy Dresden, International Journal of Particle Therapy 5(1): 172-182.

Bijl, H. P., van Luijk, P., Coppes, R. P., Schippers, J. M., Konings, A. W. \& van der Kogel, A. J. (2002). Dose-volume effects in the rat cervical spinal cord after proton irradiation, International Journal of Radiation Oncology*Biology*Physics 52(1): 205-211.

Bortfeld, T. (1997). An analytical approximation of the Bragg curve for therapeutic proton beams, Medical Physics 24(12): 2024-2033.

Buonanno, M., Grilj, V. \& Brenner, D. J. (2019). Biological effects in normal cells exposed to FLASH dose rate protons, Radiotherapy and Oncology 139: 51-55.

Carey, D. C. (1987). The Optics of Charged Particle Beams, Vol. 6, Harwood Academic Pub.

Durante, M. (2014). New challenges in high-energy particle radiobiology, The British Journal of Radiology 87(1035): 20130626.

Ford, E., Emery, R., Huff, D., Narayanan, M., Schwartz, J., Cao, N., Meyer, J., Rengan, R., Zeng, J., Sandison, G., Laramore, G. \& Mayr, N. (2017). An image-guided precision proton radiation 
platform for preclinical in vivo research, Physics in Medicine and Biology 62(1): 43-58.

Grassberger, C., Lomax, A. \& Paganetti, H. (2015). Characterizing a proton beam scanning system for Monte Carlo dose calculation in patients, Physics in Medicine and Biology 60(2): 633645.

Greubel, C., Assmann, W., Burgdorf, C., Dollinger, G., Du, G., Hable, V., Hapfelmeier, A., Hertenberger, R., Kneschaurek, P., Michalski, D., Molls, M., Reinhardt, S., Röper, B., Schell, S., Schmid, T. E., Siebenwirth, C., Wenzl, T., Zlobinskaya, O. \& Wilkens, J. J. (2011). Scanning irradiation device for mice in vivo with pulsed and continuous proton beams, Radiation and Environmental Biophysics 50(3): 339-344.

Highland, V. L. (1975). Some practical remarks on multiple scattering, Nuclear Instruments and Methods 129(2): 497-499.

Hinterberger, F. (2008). Physik der Teilchenbeschleuniger und Ionenoptik, second edn, Springer Berlin Heidelberg.

Hong, L., Goitein, M., Bucciolini, M., Comiskey, R., Gottschalk, B., Rosenthal, S., Serago, C. \& Urie, M. (1996). A pencil beam algorithm for proton dose calculations, Physics in Medicine and Biology 41(8): 1305-1330.

Kim, M. M., Irmen, P., Shoniyozov, K., Verginadis, I. I., Cengel, K. A., Koumenis, C., Metz, J. M., Dong, L. \& Diffenderfer, E. S. (2019). Design and commissioning of an image-guided small animal radiation platform and quality assurance protocol for integrated proton and x-ray radiobiology research, Physics in Medicine $\&$ Biology 64(13): 135013.

Kleeven, W., Abs, M., Forton, E., Henrotin, S., Jongen, Y., Nuttens, V., Paradis, Y., Pearson, E., Quets, S., de Walle, J. V., Verbruggen, P., Zaremba, S., Conjat, M., Mandrillon, J., Mandrillon, P. \& Development, A. (2013). The IBA Superconducting Synchrocyclotron Project S2C2, p. 5 .

Kondo, N., Sakurai, Y., Takata, T., Takai, N., Nakagawa, Y., Tanaka, H., Watanabe, T., Kume, K., Toho, T., Miyatake, S.-i., Suzuki, M., Masunaga, S.-i. \& Ono, K. (2015). Localized radiation necrosis model in mouse brain using proton ion beams, Applied Radiation and Isotopes 106: 242-246.

Kundel, S. (2019). Towards a Beamline for Small Animal Irradiation at Clinical Proton Therapy Facilities: Experimental and Computational Studies, Master's thesis, Ludwig Maximilians Universität München.

Kurichiyanil, N., Pinto, M., Rösch, T., Kundel, S., Würl, M., Englbrecht, F. S., Schreiber, J. \& Parodi, K. (2019). Design of An Adaptable Permanent-Magnet Quadrupole Triplet for Refocusing of Energy Degraded Proton Beams for Small Animal Irradiation, AAPM Annual Meeting, San Antonio TX,USA.

Liu, W., Zhang, X., Li, Y. \& Mohan, R. (2012). Robust optimization of intensity modulated proton therapy: Robust optimization of IMPT, Medical Physics 39(2): 1079-1091.

Moyers, M. F., Reder, C. S. \& Lau, D. C. (2007). Generation and Characterization of a Proton Microbeam for Experimental Radiosurgery, Technology in Cancer Research 83 Treatment 6(3): 205-211.

Müller, C., De Prado Leal, M., Dominietto, M. D., Umbricht, C. A., Safai, S., Perrin, R. L., Egloff, M., Bernhardt, P., van der Meulen, N. P., Weber, D. C., Schibli, R. \& Lomax, A. J. (2019). 
Combination of Proton Therapy and Radionuclide Therapy in Mice: Preclinical Pilot Study at the Paul Scherrer Institute, Pharmaceutics 11(9): 450.

Newhauser, W. D. \& Zhang, R. (2015). The physics of proton therapy, Phys. Med. Biol. p. 56.

Parodi, K., Assmann, W., Belka, C., Bortfeldt, J., Clevert, D.-A., Dedes, G., Kalunga, R., Kundel, S., Kurichiyanil, N., Lämmer, P., Lascaud, J., Lauber, K., Lovatti, G., Meyer, S., Nitta, M., Pinto, M., Safari, M. J., Schnürle, K., Schreiber, J., Thirolf, P. G., Wieser, H.-P. \& Würl, M. (2019). Towards a novel small animal proton irradiation platform: The SIRMIO project, Acta Oncologica pp. 1-6.

Parodi, K., Mairani, A., Brons, S., Hasch, B. G., Sommerer, F., Naumann, J., Jäkel, O., Haberer, T. \& Debus, J. (2012). Monte Carlo simulations to support start-up and treatment planning of scanned proton and carbon ion therapy at a synchrotron-based facility, Physics in Medicine and Biology 57(12): 3759-3784.

Pedroni, E., Scheib, S., Böhringer, T., Coray, A., Grossmann, M., Lin, S. \& Lomax, A. (2005). Experimental characterization and physical modelling of the dose distribution of scanned proton pencil beams, Physics in Medicine 83 Biology 50(3): 541.

Penner, S. (1961). Calculations of Properties of Magnetic Deflection Systems, Review of Scientific Instruments 32(2): 150-160.

PSI (2019). https://www.psi.ch/de/protontherapy/protonentherapie-am-psi.

PTCOG (2019). Facilities in Operation, https://www.ptcog.ch/index.php/facilities-in-operation.

Safai, S., Bortfeld, T. \& Engelsman, M. (2008). Comparison between the lateral penumbra of a collimated double-scattered beam and uncollimated scanning beam in proton radiotherapy, Physics in Medicine and Biology 53(6): 1729-1750.

Schuemann, J., Dowdell, S., Grassberger, C., Min, C. H. \& Paganetti, H. (2014). Site-specific range uncertainties caused by dose calculation algorithms for proton therapy, Physics in Medicine and Biology 59(15): 4007-4031.

Seltzer, S. M., Fernandez-Varea, J. M., Andreo, P., Bergstrom, P. M. J., Burns, D. T., Krajcar Bronic, I., Ross, C. K. \& Salvat, F. (2014). Report 90: Key data for ionizingradiation dosimetry: Measurement standards and applications, Journal of the International Commission on Radiation Units and Measurements 14(1): 1-110.

Suit, H., Phil, D., Goitein, M., Munzenrider, J., Verhey, L., Blitzer, P., Gragoudas, E., Koehler, A., Urie, M., Gentry, R., Shipley, W., Urano, M., Duttenhaver, J. \& Wagner, M. (1982). Evaluation of the clinical applicability of proton beams in definitive fractionated radiation therapy, International Journal of Radiation Oncology*Biology*Physics 8(12): 2199-2205.

Takata, T., Kondo, N., Sakurai, Y., Tanaka, H., Hasegawa, T., Kume, K. \& Suzuki, M. (2015). Reprint of Localized dose delivering by ion beam irradiation for experimental trial of establishing brain necrosis model, Applied Radiation and Isotopes 106: 104-106.

Tommasino, F., Rovituso, M., Fabiano, S., Piffer, S., Manea, C., Lorentini, S., Lanzone, S., Wang, Z., Pasini, M., Burger, W., La Tessa, C., Scifoni, E., Schwarz, M. \& Durante, M. (2017). Proton beam characterization in the experimental room of the Trento Proton Therapy facility, Nuclear Instruments and Methods in Physics Research Section A: Accelerators, Spectrometers, Detectors and Associated Equipment 869: 15-20.

van de Water, S., Safai, S., Schippers, J. M., Weber, D. C. \& Lomax, A. J. (2019). Towards 
FLASH proton therapy: The impact of treatment planning and machine characteristics on achievable dose rates, Acta Oncologica 58(10): 1463-1469.

Verhaegen, F., Dubois, L., Gianolini, S., Hill, M. A., Karger, C. P., Lauber, K., Prise, K. M., Sarrut, D., Thorwarth, D., Vanhove, C., Vojnovic, B., Weersink, R., Wilkens, J. J. \& Georg, D. (2018). ESTRO ACROP: Technology for precision small animal radiotherapy research: Optimal use and challenges, Radiotherapy and Oncology 126(3): 471-478.

Wiedemann, H. (1993). Particle Accelerator Physics, Springer Berlin Heidelberg.

Würl, M., Englbrecht, F., Parodi, K. \& Hillbrand, M. (2016). Dosimetric impact of the low-dose envelope of scanned proton beams at a ProBeam facility: Comparison of measurements with TPS and MC calculations, Physics in Medicine and Biology 61(2): 958-973. 